\title{
A New Proof of the Mullineux Conjecture
}

\author{
JONATHAN BRUNDAN \\ JONATHAN KUJAWA \\ Department of Mathematics, University of Oregon, Eugene, OR 97401, USA
}

brundan@darkwing.uoregon.edu kujawa@noether.uoregon.edu

Received May 24, 2002; Revised October 28, 2002

\begin{abstract}
Let $S_{d}$ denote the symmetric group on $d$ letters. In 1979 Mullineux conjectured a combinatorial algorithm for calculating the effect of tensoring with an irreducible $S_{d}$-module with the one dimensional sign module when the ground field has positive characteristic. Kleshchev proved the Mullineux conjecture in 1996. In the present article we provide a new proof of the Mullineux conjecture which is entirely independent of Kleshchev's approach. Applying the representation theory of the supergroup $G L(m \mid n)$ and the supergroup analogue of Schur-Weyl Duality it becomes straightforward to calculate the combinatorial effect of tensoring with the sign representation and, hence, to verify Mullineux's conjecture. Similar techniques also allow us to classify the irreducible polynomial representations of $G L(m \mid n)$ of degree $d$ for arbitrary $m, n$, and $d$.
\end{abstract}

Keywords: symmetric group, Mullineux, modular representation theory, supergroups, $G L(m \mid n)$

\section{Introduction}

Let $S_{n}$ be the symmetric group on $n$ letters, $k$ be a field of characteristic $p$ and $D^{\lambda}$ be the irreducible $k S_{n}$-module corresponding to a $p$-regular partition $\lambda$ of $n$, as in [12]. By tensoring $D^{\lambda}$ with the 1-dimensional sign representation we obtain another irreducible $k S_{n^{-}}$ module. If $p=0, D^{\lambda} \otimes \operatorname{sgn} \cong D^{\lambda^{\prime}}$, where $\lambda^{\prime}$ is the conjugate of the partition $\lambda$, and if $p=2$, we obviously have that $D^{\lambda} \otimes \operatorname{sgn} \cong D^{\lambda}$. In all other cases, it is surprisingly difficult to describe the partition labeling the irreducible module $D^{\lambda} \otimes \operatorname{sgn}$ combinatorially. In 1979, Mullineux [22] gave an algorithmic construction of a bijection M on $p$-regular partitions, and conjectured that $D^{\lambda} \otimes \operatorname{sgn} \cong D^{\mathrm{M}(\lambda)}$.

Mullineux's conjecture was finally proved in 1996 . The key breakthrough leading to the proof was made in [16], when Kleshchev discovered an alternative algorithm, quite different in nature to Mullineux's, and proved that it computes the label of $D^{\lambda} \otimes \operatorname{sgn}$. Then Ford and Kleshchev [10] proved combinatorially that Kleshchev's algorithm was equivalent to Mullineux's, hence proving the Mullineux conjecture. Since then, different and easier approaches to the combinatorial part of the proof, i.e. that Kleshchev's algorithm equals Mullineux's algorithm, have been found by Bessenrodt and Olsson [3] and by Xu [29]. Also Lascoux et al. [18] have used Ariki's theorem [1] to give a different proof of the results of [16].

The purpose of the present article is to explain a completely different proof of the Mullineux conjecture. In [28], Xu discovered yet another algorithm, and gave a short combinatorial argument to show that it was equivalent to Mullineux's original algorithm. We 
will show directly from representation theory that Xu's algorithm computes the label of $D^{\lambda} \otimes$ sgn. In this way, we obtain a relatively direct proof of the Mullineux conjecture that bypasses Kleshchev's algorithm altogether.

The idea behind our approach is a simple one. There is a superalgebra analogue of SchurWeyl duality relating representations of $S_{n}$ to representations of the supergroup $G L(n \mid n)$. Moreover, there is an involution on representations of $G L(n \mid n)$ induced by twisting with its natural outer automorphism, which corresponds under Schur-Weyl duality to tensoring with the sign representation. Ideas of Serganova [25] give an easy-to-prove algorithm for computing this involution, hence by Schur-Weyl duality we obtain an algorithm for computing $D^{\lambda} \otimes$ sgn. Actually, we obtain a whole family of algorithms, one of which turns out to be the same as Xu's algorithm.

The remainder of the article is arranged as follows. In Section 2, we review some generalities concerning the supergroup $G=G L(m \mid n)$. In Section 3, we introduce the superalgebra $\operatorname{Dist}(G)$ of distributions on $G$ and explain how integrable representations of $\operatorname{Dist}(G)$ can be lifted to $G$ itself. Serganova's algorithm is derived in Section 4 using some highest weight theory. In Section 5, we review some known results about polynomial representations and Schur-Weyl duality, allowing us to descend to the symmetric group. Finally in Section 6 we put it all together with some combinatorics to obtain the proof of the Mullineux conjecture. At the end of Section 6, we also solve a related question concerning the classification of the irreducible polynomial representations of $G L(m \mid n)$ in positive characteristic, extending work of Donkin [9]. The answer is a natural generalization of the "hook theorem" of Berele and Regev [2] and Sergeev [26] in characteristic 0.

\section{The supergroup $G L(m \mid n)$}

Throughout, let $k$ be a field of characteristic $p \neq 2$. All objects (superalgebras, supergroups, ...) will be defined over $k$. A commutative superalgebra is a $\mathbb{Z}_{2}$-graded associative algebra $A=A_{\overline{0}} \oplus A_{\overline{1}}$ with $a b=(-1)^{\bar{a} \bar{b}} b a$ for all homogeneous $a, b \in A$, where $\bar{x} \in \mathbb{Z}_{2}$ denotes the parity of a homogeneous vector $x$ in a vector superspace. For an account of the basic language of superalgebras and supergroups adopted here, we refer the reader to [6, 7], see also [13, 14, 19, Ch. I] and [20, Ch. 3, Sections 1 and 2, Ch. 4, Section 1].

The supergroup $G=G L(m \mid n)$ is the functor from the category of commutative superalgebras to the category of groups defined on a commutative superalgebra $A$ by letting $\mathrm{G}(A)$ be the group of all invertible $(m+n) \times(m+n)$ matrices of the form

$$
g=\left(\begin{array}{l|l}
W & X \\
\hline Y & Z
\end{array}\right)
$$

where $W$ is an $m \times m$ matrix with entries in $A_{\overline{0}}, X$ is an $m \times n$ matrix with entries in $A_{\overline{1}}, Y$ is an $n \times m$ with entries in $A_{\overline{1}}$, and $Z$ is an $n \times n$ matrix with entries in $A_{\overline{0}}$. If $f: A \rightarrow B$ is a superalgebra homomorphism, then $G(f): G(A) \rightarrow G(B)$ is the group homomorphism defined by applying $f$ to the matrix entries.

Let $M a t$ be the affine superscheme with $\operatorname{Mat}(A)$ consisting of all (not necessarily invertible) $(m+n) \times(m+n)$ matrices of the above form. For $1 \leq i, j \leq m+n$, let $T_{i, j}$ be the function mapping a matrix to its $i j$-entry. Then, the coordinate ring $k[M a t]$ is the free 
commutative superalgebra on the generators $\left\{T_{i, j} \mid 1 \leq i, j \leq m+n\right\}$. Writing $\bar{i}=\overline{0}$ for $i=1, \ldots, m$ and $\bar{i}=\overline{1}$ for $i=m+1, \ldots, m+n$, the parity of the generator $T_{i, j}$ is $\bar{i}+\bar{j}$. By [19, I.7.2], a matrix $g \in \operatorname{Mat}(A)$ of the form (2.1) is invertible if and only if $\operatorname{det} W \operatorname{det} Z \in A^{\times}$. Hence, $G$ is the principal open subset of Mat defined by the function $\operatorname{det}: g \mapsto \operatorname{det} W \operatorname{det} Z$. In particular, the coordinate ring $k[G]$ is the localization of $k[M a t]$ at det.

Just like for group schemes [13, I.2.3], the coordinate ring $k[G]$ has the naturally induced structure of a Hopf superalgebra. Explicitly, the comultiplication and counit of $k[G]$ are the unique superalgebra maps satisfying

$$
\begin{aligned}
\Delta\left(T_{i, j}\right) & =\sum_{h=1}^{m+n} T_{i, h} \otimes T_{h, j}, \\
\varepsilon\left(T_{i, j}\right) & =\delta_{i, j}
\end{aligned}
$$

for all $1 \leq i, j \leq m+n$. The subalgebra $k[$ Mat $]$ of $k[G]$ is a subbialgebra but not a Hopf subalgebra, as it is not invariant under the antipode.

It is sometimes convenient to work with an alternative set of generators for the coordinate ring $k[G]$ : define

$$
\tilde{T}_{i, j}=(-1)^{\bar{i}(\bar{i}+\bar{j})} T_{i, j}
$$

In terms of these new generators, (2.2) becomes

$$
\Delta\left(\tilde{T}_{i, j}\right)=\sum_{h=1}^{m+n}(-1)^{(\bar{i}+\bar{h})(\bar{h}+\bar{j})} \tilde{T}_{i, h} \otimes \tilde{T}_{h, j} .
$$

A representation of $G$ means a natural transformation $\rho: G \rightarrow G L(M)$ for some vector superspace $M$, where $G L(M)$ is the supergroup with $G L(M)(A)$ being equal to the group of all even automorphisms of the $A$-supermodule $M \otimes A$, for each commutative superalgebra $A$. Equivalently, as with group schemes [13, I.2.8], $M$ is a right $k[G]$-comodule, i.e. there is an even structure map $\eta: M \rightarrow M \otimes k[G]$ satisfying the usual comodule axioms. We will usually refer to such an $M$ as a $G$-supermodule. For example, we have the natural representation $V$, the $m \mid n$-dimensional vector superspace with canonical basis $v_{1}, \ldots, v_{m}, v_{m+1}, \ldots, v_{m+n}$ where $\bar{v}_{i}=\bar{i}$. Identify elements of $V \otimes A$ with column vectors via

$$
\sum_{i=1}^{m+n} v_{i} \otimes a_{i} \longleftrightarrow\left(\begin{array}{c}
a_{1} \\
\vdots \\
a_{m+n}
\end{array}\right) .
$$

Then, the $G(A)$-action on $V \otimes A$ is the usual one by left multiplication. The induced comodule structure map $\eta: V \rightarrow V \otimes k[G]$ is given explicitly by

$$
\eta\left(v_{j}\right)=\sum_{i=1}^{m+n} v_{i} \otimes T_{i, j}=\sum_{i=1}^{m+n}(-1)^{\bar{i}(\bar{i}+\bar{j})} v_{i} \otimes \tilde{T}_{i, j} .
$$


The underlying purely even group $G_{\mathrm{ev}}$ of $G$ is by definition the functor from superalgebras to groups with $G_{\mathrm{ev}}(A):=G\left(A_{\overline{0}}\right)$. Thus, $G_{\mathrm{ev}}(A)$ consists of all invertible matrices of the form (2.1) with $X=Y=0$, so $G_{\mathrm{ev}} \cong G L(m) \times G L(n)$. Let $T$ be the usual maximal torus of $G_{\mathrm{ev}}$ consisting of diagonal matrices. The character group $X(T)=\operatorname{Hom}\left(T, \mathbb{G}_{m}\right)$ is the free abelian group on generators $\varepsilon_{1}, \ldots, \varepsilon_{m}, \varepsilon_{m+1}, \ldots, \varepsilon_{m+n}$, where $\varepsilon_{i}$ picks out the $i$ th diagonal entry of a diagonal matrix. Put a symmetric bilinear form on $X(T)$ by declaring that

$$
\left(\varepsilon_{i}, \varepsilon_{j}\right)=(-1)^{\bar{i}} \delta_{i, j}
$$

Let $W \cong S_{m} \times S_{n}$ be the Weyl group of $G_{\mathrm{ev}}$ with respect to $T$, identified with the subgroup of $G_{\mathrm{ev}}$ consisting of all permutation matrices.

A full flag $F=\left(F_{1} \subset \cdots \subset F_{m+n}\right)$ in the vector superspace $V$ means a chain of subsuperspaces of $V$ with each $F_{i}$ having dimension $i$ as a vector space. If $\left(u_{1}, u_{2}, \ldots, u_{m+n}\right)$ is an ordered homogeneous basis for $V$, we write $F\left(u_{1}, u_{2}, \ldots, u_{m+n}\right)$ for the full flag with $F_{i}=\left\langle u_{1}, \ldots, u_{i}\right\rangle$. By definition, a Borel subgroup $B$ of $G$ is the stabilizer of a full flag $F$ in $V$, i.e. $B(A)$ is the stabilizer in $G(A)$ of the canonical image of $F$ in $V \otimes A$ for each commutative superalgebra $A$. Since $G L(m)$ (resp. $G L(n)$ ) acts transitively on the bases of $V_{\overline{0}}$ (resp. $V_{\overline{1}}$ ), it is easy to see two full flags $F$ and $F^{\prime}$ in $V$ are conjugate under $G$ if and only if the superdimension of $F_{i}$ equals the superdimension of $F_{i}^{\prime}$ for each $i=1, \ldots, m+n$. Consequently there are $\left(\begin{array}{c}m+n \\ n\end{array}\right)$ different conjugacy classes of Borel subgroups.

View the Weyl group $W$ of $G$ as the parabolic subgroup $S_{m} \times S_{n}$ of the symmetric group $S_{m+n}$ in the obvious way. Let $D_{m, n}$ be the set of all minimal length $S_{m} \times S_{n} \backslash S_{m+n}$-coset representatives, i.e.

$$
D_{m, n}=\left\{w \in S_{m+n} \mid w^{-1} 1<\cdots<w^{-1} m, w^{-1}(m+1)<\cdots<w^{-1}(m+n)\right\} .
$$

For $w \in S_{m+n}$, let $B_{w}$ be the stabilizer of the full flag $F\left(v_{w 1}, v_{w 2}, \ldots, v_{w(m+n)}\right)$. Then, the Borel subgroups $\left\{B_{w} \mid w \in D_{m, n}\right\}$ give a set of representatives for the conjugacy classes of Borel subgroup in $G$ (cf. [15, Proposition 1.2(a)]). We point out that for $w \in D_{m, n}$, the underlying even subgroup of $B_{w}$ is always the usual upper triangular Borel subgroup $B_{\mathrm{ev}}$ of $G_{\mathrm{ev}}$.

The root system of $G$ is the set $\Phi=\left\{\varepsilon_{i}-\varepsilon_{j} \mid 1 \leq i, j \leq m+n, i \neq j\right\}$. There are even and odd roots, the parity of the root $\varepsilon_{i}-\varepsilon_{j}$ being $\bar{i}+\bar{j}$. Choosing $w \in S_{m+n}$ fixes a choice $B_{w}$ of Borel subgroup of $G$ containing $T$, hence a set

$$
\Phi_{w}^{+}=\left\{\varepsilon_{w i}-\varepsilon_{w j} \mid 1 \leq i<j \leq m+n\right\}
$$

of positive roots. The corresponding dominance ordering on $X(T)$ is denoted $\leq_{w}$, defined by $\lambda \leq_{w} \mu$ if $\mu-\lambda \in \mathbb{Z}_{\geq 0} \Phi_{w}^{+}$.

For examples, first take $w=1$. Then, $B_{1}=\operatorname{stab}_{G} F\left(v_{1}, v_{2}, \ldots, v_{m+n}\right)$ is the Borel subgroup with $B_{1}(A)$ consisting of all upper triangular invertible matrices of the form (2.1). This is the standard choice of Borel subgroup, giving rise to the standard choice of positive roots $\Phi_{1}^{+}$and the standard dominance ordering $\leq_{1}$ on $X(T)$. Instead, let $w_{0}$ be the longest element of $S_{m} \times S_{n}$ and $w_{1}$ be the longest element of $D_{m, n}$, so that $w_{0} w_{1}$ is the longest 
element of the symmetric group $S_{m+n}$. Then, $B_{w_{1}}=\operatorname{stab}_{G} F\left(v_{m+1}, \ldots, v_{m+n}, v_{1}, \ldots, v_{m}\right)$ is the Borel with $B_{w_{1}}(A)$ consisting of all invertible matrices of the form (2.1) with $X=0$ and $W, Z$ upper triangular. Finally, $B_{w_{0} w_{1}}=w_{0} B_{w_{1}} w_{0}^{-1}$ is the Borel subgroup of all lower triangular matrices.

\section{The superalgebra of distributions}

We next recall the definition of the superalgebra of distributions $\operatorname{Dist}(G)$ of $G$, following [6, Section 4]. Let $\mathcal{I}_{1}$ be the kernel of the counit $\varepsilon: k[G] \rightarrow k$, a superideal of $k[G]$. For $r \geq 0$, let

$$
\begin{aligned}
\operatorname{Dist}_{r}(G) & =\left\{x \in k[G]^{*} \mid x\left(\mathcal{I}_{1}^{r+1}\right)=0\right\} \cong\left(k[G] / \mathcal{I}_{1}^{r+1}\right)^{*} \\
\operatorname{Dist}(G) & =\bigcup_{r \geq 0} \operatorname{Dist}_{r}(G)
\end{aligned}
$$

There is a multiplication on $k[G]^{*}$ dual to the comultiplication on $k[G]$, defined by $(x y)(f)=$ $(x \bar{\otimes} y)(\Delta(f))$ for $x, y \in k[G]^{*}$ and $f \in k[G]$. Note here (and later on) we are implicitly using the superalgebra rule of signs: $(x \bar{\otimes} y)(f \otimes g)=(-1)^{\bar{y}} \bar{f} x(f) y(g)$. One can check that $\operatorname{Dist}(G)$ is a subsuperalgebra of $k[G]^{*}$ using the fact that for $f \in \mathcal{I}_{1}$,

$$
\Delta(f) \in 1 \otimes f+f \otimes 1+\mathcal{I}_{1} \otimes \mathcal{I}_{1}
$$

or, more generally,

$$
\Delta\left(f_{1} \ldots f_{r}\right) \in \prod_{i=1}^{r}\left(1 \otimes f_{i}+f_{i} \otimes 1\right)+\sum_{j=1}^{r} \mathcal{I}_{1}^{j} \otimes \mathcal{I}_{1}^{r+1-j}
$$

for all $f_{1}, \ldots, f_{r} \in \mathcal{I}_{1}$. In fact, since $\mathcal{I}_{1}^{r+1} \subseteq \mathcal{I}_{1}^{r}$, we have $\operatorname{Dist}_{r}(G) \subseteq \operatorname{Dist}_{r+1}(G)$ and (3.1) shows that $\operatorname{Dist}_{r}(G) \operatorname{Dist}_{s}(G) \subseteq \operatorname{Dist}_{r+s}(G)$, i.e. $\operatorname{Dist}(G)$ is a filtered superalgebra. By (3.1) again, the subspace

$$
T_{1}(G)=\left\{x \in \operatorname{Dist}_{1}(G) \mid x(1)=0\right\} \cong\left(\mathcal{I}_{1} / \mathcal{I}_{1}^{2}\right)^{*}
$$

is closed under the superbracket $[x, y]:=x y-(-1)^{\bar{x} \bar{y}} y x$, giving $T_{1}(G)$ the structure of Lie superalgebra, denoted $\operatorname{Lie}(G)$. Finally, given a $G$-supermodule $M$ with structure map $\eta: M \rightarrow M \otimes k[G]$, we can view $M$ as a $\operatorname{Dist}(G)$-supermodule by $x . m=(1 \bar{\otimes} x)(\eta(m))$. In particular, this makes $M$ into a $\operatorname{Lie}(G)$-supermodule.

To describe $\operatorname{Lie}(G)$ explicitly in our case, recall the alternative generators $\tilde{T}_{i, j}$ of $k[G]$ from (2.4). The superideal $\mathcal{I}_{1}$ is generated by $\left\{\tilde{T}_{i, j}-\delta_{i, j} \mid 1 \leq i, j \leq m+n\right\}$. So Lie $(G)$ has a unique basis $\left\{e_{i, j} \mid 1 \leq i, j \leq m+n\right\}$ such that $e_{i, j}\left(\tilde{T}_{h, l}\right)=\delta_{i, h} \delta_{j, l}$. The parity of $e_{i, j}$ is $\bar{i}+\bar{j}$, while (2.2) implies that the multiplication satisfies

$$
\left[e_{i, j}, e_{h, l}\right]=\delta_{j, h} e_{i, l}-(-1)^{(\bar{i}+\bar{j})(\bar{h}+\bar{l})} \delta_{i, l} e_{h, j}
$$


Thus $\operatorname{Lie}(G)$ is identified with the Lie superalgebra $\mathfrak{g l}(m \mid n)$ over $k$, see [14], so that $e_{i, j}$ corresponds to the $i j$-matrix unit. By (2.6), the induced action of $\operatorname{Lie}(G)$ on the natural representation $V$ of $G$ is given by $e_{i, j} v_{h}=\delta_{j, h} v_{i}$, i.e. $V$ is identified with the natural representation of $\mathfrak{g l}(m \mid n)$.

To describe $\operatorname{Dist}(G)$ explicitly, first note that over $\mathbb{C}$, Dist $(G)$ is simply the universal enveloping superalgebra of $\operatorname{Lie}(G)$. To construct $\operatorname{Dist}(G)$ in general, let $U_{\mathbb{C}}$ be the universal enveloping superalgebra of the Lie superalgebra $\mathfrak{g l}(m \mid n)$ over $\mathbb{C}$. By the PBW theorem for Lie superalgebras (see [14]), $U_{\mathbb{C}}$ has basis consisting of all monomials

$$
\prod_{\substack{1 \leq i, j \leq m+n \\ \bar{i}+\bar{j}=\overline{0}}} e_{\substack{i, j \\ a_{i, j}}} \prod_{\substack{1 \leq i, j \leq m+n \\ \bar{i}+\bar{j}=\overline{1}}} e_{i, j}^{d_{i, j}}
$$

where $a_{i, j} \in \mathbb{Z}_{\geq 0}, d_{i, j} \in\{0,1\}$, and the product is taken in any fixed order. We shall write $h_{i}=e_{i, i}$ for short.

Define the Kostant $\mathbb{Z}$-form $U_{\mathbb{Z}}$ to be the $\mathbb{Z}$-subalgebra of $U_{\mathbb{C}}$ generated by elements $e_{i, j}(1 \leq i, j \leq m+n, \bar{i}+\bar{j}=\overline{1}), e_{i, j}^{(r)}(1 \leq i \neq j \leq m+n, \bar{i}+\bar{j}=\overline{0}, r \geq 1)$ and $\left(\begin{array}{c}h_{i} \\ r\end{array}\right)(1 \leq i \leq m+n, r \geq 1)$. Here, $e_{i, j}^{(r)}:=e_{i, j}^{r} /(r !)$ and $\left(\begin{array}{c}h_{i} \\ r\end{array}\right):=h_{i}\left(h_{i}-1\right) \ldots\left(h_{i}-r+1\right) /$ $(r !)$. Following the proof of [27, Th. 2], one verifies the following:

Lemma 3.1 The superalgebra $U_{\mathbb{Z}}$ is a $\mathbb{Z}$-free $\mathbb{Z}$-module with basis being given by the set of all monomials of the form

$$
\prod_{\substack{1 \leq i, j \leq m+n \\
\bar{i}+\bar{j}=\overline{0} \\
i \neq j}} e_{i, j}^{\left(a_{i, j}\right)} \prod_{1 \leq i \leq m+n}\left(\begin{array}{c}
h_{i} \\
r_{i}
\end{array}\right) \prod_{\substack{1 \leq i, j \leq m+n \\
\bar{i}+\bar{j}=\overline{1}}} e_{i, j}^{d_{i, j}}
$$

for all $a_{i, j}, r_{i} \in \mathbb{Z}_{\geq 0}$ and $d_{i, j} \in\{0,1\}$, where the product is taken in any fixed order.

The enveloping superalgebra $U_{\mathbb{C}}$ is a Hopf superalgebra in a canonical way, hence $U_{\mathbb{Z}}$ is a Hopf superalgebra over $\mathbb{Z}$. Finally, set $U_{k}=k \otimes_{\mathbb{Z}} U_{\mathbb{Z}}$, naturally a Hopf superalgebra over $k$. We will abuse notation by using the same symbols $e_{i, j}^{(r)},\left(\begin{array}{c}h_{i} \\ r\end{array}\right)$ etc ... for the canonical images of these elements of $U_{\mathbb{Z}}$ in $U_{k}$. Now the basic fact is the following:

Theorem 3.2 $U_{k}$ and $\operatorname{Dist}(G)$ are isomorphic as Hopf superalgebras.

Proof: In the case when $k=\mathbb{C}$, the isomorphism $i: U_{\mathbb{C}} \rightarrow \operatorname{Dist}(G)$ is induced by the Lie superalgebra isomorphism mapping the matrix unit $e_{i, j} \in \mathfrak{g l}(m \mid n)$ to the element with the same name in $\operatorname{Lie}(G)$. For arbitrary $k$, the isomorphism $i: U_{k} \rightarrow \operatorname{Dist}(G)$ is obtained by reducing this one modulo $p$.

In view of the theorem, we will henceforth identify $U_{k}$ with $\operatorname{Dist}(G)$. It is also easy to describe the superalgebras of distributions of our various natural subgroups of $G$ as subalgebras of $\operatorname{Dist}(G)$. For example, $\operatorname{Dist}(T)$ is the subalgebra generated by all $\left(\begin{array}{c}h_{i} \\ r\end{array}\right)(1 \leq$ $i \leq m+n, r \geq 1), \operatorname{Dist}\left(B_{\mathrm{ev}}\right)$ is the subalgebra generated by $\operatorname{Dist}(T)$ and all $e_{i, j}^{(r)}(1 \leq i<$ 
$j \leq m+n, \bar{i}+\bar{j}=\overline{0}, r \geq 1)$, and for $w \in D_{m, n}, \operatorname{Dist}\left(B_{w}\right)$ is the subalgebra generated by $\operatorname{Dist}\left(B_{\text {ev }}\right)$ and all $e_{i, j}\left(1 \leq \bar{i}, j \leq m+n, \bar{i}+\bar{j}=1, w^{-1} i<w^{-1} j\right)$.

For $\lambda=\sum_{i=1}^{m+n} \lambda_{i} \varepsilon_{i} \in X(T)$ and a $\operatorname{Dist}(G)$-supermodule $M$, define the $\lambda$-weight space of $M$ to be

$$
M_{\lambda}=\left\{m \in M \mid\left(\begin{array}{c}
h_{i} \\
r
\end{array}\right) m=\left(\begin{array}{c}
\lambda_{i} \\
r
\end{array}\right) m \text { for all } i=1, \ldots, m+n, r \geq 1\right\} .
$$

We call a $\operatorname{Dist}(G)$-supermodule $M$ integrable if it is locally finite over $\operatorname{Dist}(G)$ and satisfies $M=\sum_{\lambda \in X(T)} M_{\lambda}$. If $M$ is a $G$-supermodule viewed as a $\operatorname{Dist}(G)$-supermodule in the natural way, then $M$ is integrable. The goal in the remainder of the section is to prove conversely that any integrable $\operatorname{Dist}(G)$-supermodule can be lifted in a unique way to $G$.

Let $\operatorname{Dist}(G)^{\diamond}$ denote the restricted dual of $\operatorname{Dist}(G)$, namely, the set of all $f \in \operatorname{Dist}(G)^{*}$ such that $f(I)=0$ for some two-sided superideal $I \subset \operatorname{Dist}(G)$ (depending on $f$ ) with $\operatorname{Dist}(G) / I$ being a finite dimensional integrable $\operatorname{Dist}(G)$-supermodule. If $M$ is an integrable $\operatorname{Dist}(G)$-supermodule with homogenous basis $\left\{m_{i}\right\}_{i \in I}$, its coefficient space $c f(M)$ is the subspace of $\operatorname{Dist}(G)^{*}$ spanned by the coefficient functions $f_{i, j}$ defined by

$$
u m_{j}=(-1)^{\bar{u} \bar{m}_{j}} \sum_{i \in I} f_{i, j}(u) m_{i}
$$

For all homogeneous $u \in \operatorname{Dist}(G)$. Note that this definition is independent of the choice of homogenous basis. As in the purely even case [8, (3.1a)], we have the following lemma:

Lemma 3.3 $f \in \operatorname{Dist}(G)^{*}$ belongs to $\operatorname{Dist}(G)^{\diamond}$ if and only if $f \in c f(M)$ for some integrable $\operatorname{Dist}(G)$-supermodule $M$.

If $M$ and $N$ are integrable $\operatorname{Dist}(G)$-supermodules, then $M \otimes N$ is also an integrable supermodule and $c f(M \otimes N)=c f(M) c f(N)$. Consequently, Lemma 3.3 implies $\operatorname{Dist}(G)^{\diamond}$ is a subsuperalgebra of $\operatorname{Dist}(G)^{*}$. Indeed, $\operatorname{Dist}(G)^{\diamond}$ has a natural Hopf superalgebra structure dual to that on $\operatorname{Dist}(G)$, cf. the argument after [6, Lemma 5.2].

Theorem 3.4 The map $\iota: k[G] \rightarrow \operatorname{Dist}(G)^{\diamond}$ defined by $\iota(f)(u)=(-1)^{\bar{f}_{\bar{u}}} u(f)$ for all homogeneous $f \in k[G]$ and $u \in \operatorname{Dist}(G)$ is an isomorphism of Hopf superalgebras.

Proof: Note $\iota$ is automatically a Hopf superalgebra homomorphism, since the Hopf superalgebra structure on $\operatorname{Dist}(G)$ is dual to that on $k[G]$ and the Hopf superalgebra structure on $\operatorname{Dist}(G)^{\diamond}$ is dual to that on $\operatorname{Dist}(G)$. Furthermore if $\iota(f)=0$ then $u(f)=0$ for all $u \in \operatorname{Dist}_{r}(G)$, so $f \in \mathcal{I}_{1}^{r+1}$. Since $r$ was arbitrary we deduce $f \in \bigcap_{r \geq 0} \mathcal{I}_{1}^{r+1}$, hence $f=0$. This shows that $\iota$ is injective. It remains to prove that $\iota$ is surjective.

Fix an order for the products in the monomials in the PBW basis for $\operatorname{Dist}(G)$ from Lemma 3.1 so that all monomials are of the form $m u$ where $m$ is a monomial in the $e_{i, j}$ with $\bar{i}+\bar{j}=1$ and $u \in \operatorname{Dist}\left(G_{\mathrm{ev}}\right)$. Let $\Gamma=\{(i, j): 1 \leq i, j \leq m+n, \bar{i}+\bar{j}=\overline{1}\}$. For each $I \subseteq \Gamma$, let $m_{I}$ denote the PBW monomial given by taking the product of the $e_{i, j}$ 's for 
$(i, j) \in I$ in the fixed order. By Lemma 3.1 we have the vector space decomposition

$$
\operatorname{Dist}(G)=\bigoplus_{I \subseteq \Gamma} m_{I} \operatorname{Dist}\left(G_{\mathrm{ev}}\right) .
$$

For $I \subseteq \Gamma$, let $\eta_{I} \in \operatorname{Dist}(G)^{*}$ be the linear functional given by $\eta_{I}\left(m_{I}\right)=1$ and $\eta_{I}(m)=0$ for any other ordered PBW monomial different from $m_{I}$.

Claim 1 For any $I \subseteq \Gamma$, we have that $\eta_{I} \in \iota(k[G]) \subseteq \operatorname{Dist}(G)^{\diamond}$.

To prove this, let $N=m^{2}+n^{2}$. Let $M$ denote $\bigwedge^{N}\left(V \otimes V^{*}\right)$ viewed as a $\operatorname{Dist}(G)$ supermodule in the natural way. Since $M$ is in fact a $G$-supermodule, we have that $c f(M) \subseteq$ $\iota(k[G])$. Therefore to prove Claim 1, it suffices to show that $\eta_{I} \in c f(M)$ for any $I \subseteq \Gamma$. Let $f_{1}, \ldots, f_{m+n}$ be the basis for $V^{*}$ dual to the basis $v_{1}, \ldots, v_{m+n}$ of $V$. Let $z_{i, j}=v_{i} \otimes f_{j} \in$ $V \otimes V^{*}$. Fix a total order on the set $\{1, \ldots, m+n\} \times\{1, \ldots, m+n\}$ and in this order let $\Sigma$ be the set of all weakly increasing sequences $\left.S=\left(\left(i_{1}, j_{1}\right)\right) \leq \cdots \leq\left(i_{N}, j_{N}\right)\right)$ of length $N$ such that $\left(i_{k}, j_{k}\right)<\left(i_{k+1}, j_{k+1}\right)$ whenever $\bar{i}_{k}+\bar{j}_{k}=\overline{0}$. For $S \in \Sigma$, let $z_{S}=z_{i_{1}, j_{1}} \wedge \cdots \wedge z_{i_{N}, j_{N}}$, so that $\left\{z_{S}\right\}_{S \in \Sigma}$ is a basis for $M$. In particular, let $z=z_{S}$ for the sequence $S$ containing all $(i, j)$ with $\bar{i}+\bar{j}=\overline{0}$. Then $z$ spans $\bigwedge^{N}\left(\left(V \otimes V^{*}\right)_{\overline{0}}\right)=\bigwedge^{N}\left(V_{\overline{0}} \otimes V_{\overline{0}}^{*} \oplus V_{\overline{1}} \otimes V_{\overline{1}}^{*}\right)$, which is a 1-dimensional trivial $\operatorname{Dist}\left(G_{\mathrm{ev}}\right)$-submodule of $M$.

Observe now that $\left\{m_{I} z\right\}_{I \subseteq \Gamma}$ is a linearly independent set of homogeneous vectors, because they are related to the basis elements $\left\{z_{S}\right\}_{S \in \Sigma}$ in a unitriangular way. Extend this set to a homogeneous basis $\mathscr{B}$ of $M$. For $I \subseteq \Gamma$ and $u \in \operatorname{Dist}(G)$ define $g_{I}(u)$ to be the $m_{I} z$ coefficent of $u z$ when expressed in the basis $\mathscr{B}$. Then $g_{I}\left(m_{J}\right)=\delta_{I, J}$ for all $I, J \subseteq \Gamma$. Furthermore, since $z$ spans a trivial $\operatorname{Dist}\left(G_{\mathrm{ev}}\right)$-module, $u z=0$ for all monomials in our ordered PBW basis for $\operatorname{Dist}(G)$ not of the form $m_{J}$, i.e. $g_{I}(u)=0$ for all such monomials. Therefore $\eta_{I}=g_{I} \in c f(M)$, proving the claim.

Claim 2 For any $I \subseteq \Gamma$ and $f \in \operatorname{Dist}\left(G_{\mathrm{ev}}\right)^{\diamond}$, we have that $\left(\eta_{I} f\right)\left(m_{I} u\right)=f(u)$ and $\left(\eta_{I} f\right)\left(m_{J} u\right)=0$ for all $u \in \operatorname{Dist}\left(G_{\mathrm{ev}}\right)$ and $J \nsupseteq I$.

Indeed, by the definition of multiplication in $\operatorname{Dist}(G)^{\diamond}$, we have $\left(\eta_{I} f\right)\left(m_{J} u\right)=\left(\eta_{I} \bar{\otimes} f\right)$ $\left(\delta\left(m_{J} u\right)\right)$, where $\delta$ is the comultiplication on $\operatorname{Dist}(G)$. Recalling that $\delta\left(e_{i, j}\right)=e_{i, j} \otimes 1+$ $1 \otimes e_{i, j}$, we see that, when expressed in the ordered PBW basis of $\operatorname{Dist}(G) \otimes \operatorname{Dist}(G)$, the $\left(m_{I} \otimes-\right)$-component of $\delta\left(m_{J} u\right)$ is equal to $m_{I} \otimes u$ if $J=I$ and 0 if $J \nsupseteq I$. This implies the claim.

Claim 3 For any $f \in \operatorname{Dist}(G)^{\diamond}$ and $I \subseteq \Gamma$, there is a function $f_{I} \in \iota(k[G])$ such that $f_{I}=f$ on $m_{I} \operatorname{Dist}\left(G_{\mathrm{ev}}\right)$ and $f_{I}=0$ on $\bigoplus_{j \nsupseteq I} m_{J} \operatorname{Dist}\left(G_{\mathrm{ev}}\right)$.

To prove this, we need to appeal to the analogous theorem for the underlying even group $G_{\text {ev }}$. Just as for $\operatorname{Dist}(G)$ we can define integrable $\operatorname{Dist}\left(G_{\text {ev }}\right)$-supermodules, coefficent space, the restricted dual $\operatorname{Dist}\left(G_{\mathrm{ev}}\right)^{\diamond}$, etc... By the purely even theory, the natural map $\iota_{\mathrm{ev}}: k\left[G_{\mathrm{ev}}\right] \rightarrow \operatorname{Dist}\left(G_{\mathrm{ev}}\right)^{\diamond}$ (the analogue of the map $\iota: k[G] \rightarrow \operatorname{Dist}(G)^{\diamond}$ being considered here) is an isomorphism, see e.g. [8, (3.1c)] for the proof. An integrable $\operatorname{Dist}(G)$ supermodule is integrable over $\operatorname{Dist}\left(G_{\text {ev }}\right)$ too, so restriction gives us a Hopf superalgebra homomorphism $\vartheta: \operatorname{Dist}(G)^{\diamond} \rightarrow \operatorname{Dist}\left(G_{\mathrm{ev}}\right)^{\diamond}$ such that $\vartheta \circ \iota=\iota_{\mathrm{ev}} \circ \varphi$, where $\varphi: k[G] \rightarrow k\left[G_{\mathrm{ev}}\right]$ is the canonical map induced by the inclusion of $G_{\text {ev }}$ into $G$. 
Now take $f \in \operatorname{Dist}(G)^{\diamond}$ and write $\delta(f)=\sum_{j} f_{j} \otimes g_{j}$. By the previous paragraph, we can find even elements $h_{j} \in \iota(k[G])$ such that $\vartheta\left(g_{j}\right)=\vartheta\left(h_{j}\right)$ for each $j$. For $I \subseteq \Gamma$, let $f_{I}=\sum_{j} f_{j}\left(m_{I}\right) \eta_{I} h_{j}$, an element of $\iota(k[G])$ by Claim 1. By Claim 2, we have $f_{I}=f$ on $m_{I} \operatorname{Dist}\left(G_{\mathrm{ev}}\right)$ and $f_{I}=0$ on $\sum_{J \not \supset I} m_{J} \operatorname{Dist}\left(G_{\mathrm{ev}}\right)$, as required to prove the claim.

Now we can complete the proof. Fix $f \in \operatorname{Dist}(G)^{\diamond}$. For $i=0,1, \ldots, 2 m n$ define $f^{(i)}$ recursively by

$$
f^{(0)}=f-f_{\emptyset} \quad f^{(i)}=f^{(i-1)}-\sum_{I \subseteq \Gamma,|I|=i}\left(f^{(i-1)}\right)_{I},
$$

invoking Claim 3. An easy induction on $i$ using Claim 3 shows that $f^{(i)}=0$ on $\bigoplus_{J \subseteq \Gamma,|J| \leq i}$ $m_{J} \operatorname{Dist}\left(G_{\mathrm{ev}}\right)$. In particular, $f^{(2 m n)}=0$ on $\operatorname{Dist}(G)$. This implies the surjectivity of $\iota$, since $f$ is obtained from $f^{(2 m n)}$ by adding elements of $\iota(k[G])$.

Corollary 3.5 The category of $G$-supermodules is isomorphic to the category of integrable $\operatorname{Dist}(G)$-supermodules.

Proof: Say $M$ is an integrable $\operatorname{Dist}(G)$-supermodule with homogenous basis $\left\{m_{i}\right\}_{i \in I}$. Let $f_{i, j}$ be the corresponding coefficent functions defined according to (3.4). By Theorem 3.4, there are unique $g_{i, j} \in k[G]$ such that $\iota\left(g_{i, j}\right)=f_{i, j}$. Define a structure map $\eta: M \rightarrow$ $M \otimes k[G]$ making $M$ into a $G$-supermodule by

$$
\eta\left(m_{j}\right)=\sum_{i \in I} m_{i} \otimes g_{i, j} .
$$

Conversely, as discussed at the beginning of the section, any $G$-supermodule can be viewed as an integrable $\operatorname{Dist}(G)$-supermodule in a natural way. One can verify that these two constructions give mutually inverse functors between the two categories.

In view of the corollary, we will not distinguish between $G$-supermodules and integrable $\operatorname{Dist}(G)$-supermodules in the rest of the article.

\section{Highest weight theory}

Now we describe the classification of the irreducible representations of $G$ by their highest weights. It seems to be more convenient to work first in the category $\mathcal{O}$ of all $\operatorname{Dist}(G)$ supermodules $M$ that are locally finite over $\operatorname{Dist}\left(B_{\mathrm{ev}}\right)$ and satisfy $M=\bigoplus_{\lambda \in X(T)} M_{\lambda}$. Fix a choice of $w \in D_{m, n}$, hence a Borel subgroup $B_{w}$ and dominance ordering $\leq_{w}$ on $X(T)$. By Lemma 3.1, $\operatorname{Dist}\left(B_{w}\right)$ is a free right $\operatorname{Dist}\left(B_{\mathrm{ev}}\right)$-module of finite rank. So the condition that $M$ is locally finite over $\operatorname{Dist}\left(B_{\text {ev }}\right)$ in the definition of category $\mathcal{O}$ is equivalent to $M$ being locally finite over $\operatorname{Dist}\left(B_{w}\right)$. For $\lambda \in X(T)$, we have the Verma module

$$
M_{w}(\lambda):=\operatorname{Dist}(G) \otimes_{\operatorname{Dist}\left(B_{w}\right)} k_{\lambda},
$$

where $k_{\lambda}$ denotes $k$ viewed as a $\operatorname{Dist}\left(B_{w}\right)$-supermodule of weight $\lambda$. We say that a vector $v$ in a $\operatorname{Dist}(G)$-supermodule $M$ is a w-primitive vector of weight $\lambda$ if $\operatorname{Dist}\left(B_{w}\right) v \cong k_{\lambda}$ as a 
$\operatorname{Dist}\left(B_{w}\right)$-supermodule. Familiar arguments exactly as for semisimple Lie algebras over $\mathbb{C}$ show:

Lemma 4.1 Let $w \in D_{m, n}$ and $\lambda \in X(T)$.

(i) The $\lambda$-weight space of $M_{w}(\lambda)$ is 1-dimensional, and all other weights of $M_{w}(\lambda)$ are $<_{w} \lambda$.

(ii) Any non-zero quotient of $M_{w}(\lambda)$ is generated by a w-primitive vector of weight $\lambda$, unique up to scalars.

(iii) Any $\operatorname{Dist}(G)$-supermodule generated by a w-primitive vector of weight $\lambda$ is isomorphic to a quotient of $M_{w}(\lambda)$.

(iv) $M_{w}(\lambda)$ has a unique irreducible quotient $L_{w}(\lambda)$, and the $\left\{L_{w}(\lambda)\right\}_{\lambda \in X(T)}$ give a complete set of pairwise non-isomorphic irreducibles in $\mathcal{O}$.

In this way, we get a parametrization of the irreducible objects in $\mathcal{O}$ by their highest weights with respect to the ordering $\leq_{w}$. Of course, the parametrization depends on the initial choice of $w \in D_{m, n}$. To translate between labelings arising from different choices $w, w^{\prime} \in D_{m, n}$, it suffices to consider the situation that $w, w^{\prime}$ are adjacent with respect to the usual Bruhat ordering on $D_{m, n}$. In that case the following theorem of Serganova [25], see also [23, Lemma 0.3], does the job. For the statement, recall the definition of the form (., .) on $X(T)$ from $(2.7)$.

Lemma 4.2 Let $\lambda \in X(T)$. Suppose that $w, w^{\prime} \in D_{m, n}$ are adjacent in the Bruhat ordering, so $\Phi_{w^{\prime}}^{+}=\Phi_{w}^{+}-\{\alpha\} \cup\{-\alpha\}$ for some odd root $\alpha=\varepsilon_{i}-\varepsilon_{j} \in \Phi$. Then,

$$
L_{w}(\lambda) \cong\left\{\begin{array}{lll}
L_{w^{\prime}}(\lambda) & \text { if }(\lambda, \alpha) \equiv 0 & (\bmod p) \\
L_{w^{\prime}}(\lambda-\alpha) & \text { if }(\lambda, \alpha) \not \equiv 0 & (\bmod p)
\end{array}\right.
$$

Proof: $\quad$ Let $v$ be a $w$-primitive vector in $L_{w}(\lambda)$ of weight $\lambda$, cf. Lemma 4.1. We claim first that $e_{r, s} e_{j, i} v=0$ for all $1 \leq r, s \leq m+n$ with $\varepsilon_{r}-\varepsilon_{s} \in \Phi_{w}^{+} \cap \Phi_{w^{\prime}}^{+}$. We know that $e_{r, s} v=0$ as $v$ is $w$-primitive. So we are done immediately if $\left[e_{r, s}, e_{j, i}\right]=0$. In view of (3.2), this just leaves the possibilities $s=j$ or $r=i$. Suppose first that $s=j$. Noting that $w^{\prime}=(i j) w$, the assumption that $\varepsilon_{r}-\varepsilon_{j} \in \Phi_{w^{\prime}}^{+}$implies by (2.8) that $\varepsilon_{r}-\varepsilon_{i} \in \Phi_{w}^{+}$, hence $e_{r, i} v=0$. Therefore $e_{r, j} e_{j, i} v=e_{r, i} v=0$. The remaining case when $r=i$ is similar.

Now suppose that $e_{j, i} v \neq 0$. Since $e_{j, i}^{2}=0$, we get from the previous paragraph that $e_{j, i} v$ is $w^{\prime}$-primitive of weight $\lambda-\alpha$. Hence, $L_{w}(\lambda) \cong L_{w^{\prime}}(\lambda-\alpha)$. On the other hand, if $e_{j, i} v=0$, then $v$ itself is already $w^{\prime}$-primitive of weight $\lambda$ so $L_{w}(\lambda) \cong L_{w^{\prime}}(\lambda)$. Thus, to complete the proof of the lemma, it suffices to show that $e_{j, i} v \neq 0$ if and only if $(\lambda, \alpha) \not \equiv 0(\bmod p)$. But $e_{j, i} v \neq 0$ if and only if there is some element $x \in \operatorname{Dist}\left(B_{w}\right)$ such that $x e_{j, i} v$ is a non-zero multiple of $v$. In view of the first paragraph, the only $x$ that needs to be considered is $e_{i, j}$. Finally, $e_{i j} e_{j i} v=(-1)(\lambda, \alpha) v$.

Recall that $w_{1}$ denotes the longest element of $D_{m, n}$. For $\lambda \in X(T)$, define $\tilde{\lambda} \in X(T)$ from the isomorphism

$$
L_{1}(\lambda) \cong L_{w_{1}}(\tilde{\lambda})
$$


Lemma 4.2 implies the following algorithm for computing $\tilde{\lambda}$ :

Theorem 4.3 Pick an ordering $\beta_{1}, \ldots, \beta_{m n}$ of the roots $\left\{\varepsilon_{i}-\varepsilon_{j} \mid 1 \leq i \leq m, m+1 \leq\right.$ $j \leq m+n\}$ such that $\beta_{i} \leq{ }_{1} \beta_{j}$ implies $i \leq j$. Set $\lambda^{(0)}=\lambda$, and inductively define

$$
\lambda^{(i)}=\left\{\begin{array}{lll}
\lambda^{(i-1)} & \text { if }\left(\lambda^{(i-1)}, \beta_{i}\right) \equiv 0 & (\bmod p), \\
\lambda^{(i-1)}-\beta_{i} & \text { if }\left(\lambda^{(i-1)}, \beta_{i}\right) \neq 0 & (\bmod p),
\end{array}\right.
$$

for $i=1, \ldots, m n$. Then, $\tilde{\lambda}=\lambda^{(m n)}$.

We refer to the algorithm for $\tilde{\lambda}$ given by the theorem as Serganova's algorithm. For an example, suppose $m=n=2, p=3$ and $\lambda=\varepsilon_{1}+\varepsilon_{2}+2 \varepsilon_{3}$. Taking $\beta_{1}=\varepsilon_{2}-\varepsilon_{3}, \beta_{2}=$ $\varepsilon_{2}-\varepsilon_{4}, \beta_{3}=\varepsilon_{1}-\varepsilon_{3}, \beta_{4}=\varepsilon_{1}-\varepsilon_{4}$, we get successively $\lambda^{(1)}=\varepsilon_{1}+\varepsilon_{2}+2 \varepsilon_{3}, \lambda^{(2)}=$ $\varepsilon_{1}+2 \varepsilon_{3}+\varepsilon_{4}, \lambda^{(3)}=\varepsilon_{1}+2 \varepsilon_{3}+\varepsilon_{4}, \lambda^{(4)}=2 \varepsilon_{3}+2 \varepsilon_{4}$. Hence, $\tilde{\lambda}=2 \varepsilon_{3}+2 \varepsilon_{4}$.

Now we pass from $\mathcal{O}$ to the finite dimensional irreducible representations of $G$. We will work now just with the standard choice of Borel subgroup $B_{1}$ and the corresponding standard dominance ordering $\leq_{1}$ on $X(T)$. Let

$$
X^{+}(T)=\left\{\lambda=\sum_{i=1}^{m+n} \lambda_{i} \varepsilon_{i} \in X(T) \mid \lambda_{1} \geq \cdots \geq \lambda_{m}, \lambda_{m+1} \geq \cdots \geq \lambda_{m+n}\right\}
$$

denote the set of all dominant integral weights. The proof of the first part of the following lemma goes back to Kac [15], while the second part is due to Serganova.

Lemma 4.4 Given any $\lambda \in X(T), L_{1}(\lambda)$ is finite dimensional if and only if $\lambda \in X^{+}(T)$. Moreover, for $\lambda \in X^{+}(T)$, the $\leq_{1}$-lowest weight of $L_{1}(\lambda)$ is $w_{0} \tilde{\lambda}$.

Proof: Suppose first that $L_{1}(\lambda)$ is finite dimensional for $\lambda \in X(T)$. Then, it contains a $\operatorname{Dist}\left(B_{\mathrm{ev}}\right)$-primitive vector of weight $\lambda$, hence by the purely even theory we must have that $\lambda \in X^{+}(T)$. Conversely, suppose that $\lambda \in X^{+}(T)$. Then, there is a finite dimensional irreducible $\operatorname{Dist}\left(G_{\mathrm{ev}}\right)$-supermodule $L_{\mathrm{ev}}(\lambda)$ of highest weight $\lambda$. Let $P$ be the closed subgroup of $G$ with $P(A)$ consisting of all invertible matrices of the form (2.1) with $Y=0$. We can view $L_{\mathrm{ev}}(\lambda)$ as a $\operatorname{Dist}(P)$-supermodule so that all $e_{i, j}$ for $1 \leq i \leq m, m+1 \leq j \leq m+n$ act as zero. Consider the induced supermodule

$$
\operatorname{Dist}(G) \otimes_{\operatorname{Dist}(P)} L_{\mathrm{ev}}(\lambda)
$$

It is a finite dimensional module generated by a 1 -primitive vector of weight $\lambda$. Hence, $M_{1}(\lambda)$ has a finite dimensional quotient. This shows that $L_{1}(\lambda)$ is finite dimensional. Finally, by (4.1), $L_{1}(\lambda) \cong L_{w_{1}}(\tilde{\lambda})$. Hence, all its weights are $\leq_{w_{1}} \tilde{\lambda}$. Since $L_{1}(\lambda)$ is finite dimensional, the Weyl group $W$ acts by permuting weights. Hence we can act with $w_{0}$ to get that all its weights are $\geq_{1} w_{0} \tilde{\lambda}$.

Lemmas 4.1 and 4.4 show that $\left\{L_{1}(\lambda)\right\}_{\lambda \in X^{+}(T)}$ is a complete set of pairwise non-isomorphic irreducible integrable $\operatorname{Dist}(G)$-supermodules. In view of Corollary 3.5, we can lift the 
$\operatorname{Dist}(G)$-supermodule $L_{1}(\lambda)$ for $\lambda \in X^{+}(T)$ uniquely to $G$. We will denote the resulting irreducible $G$-supermodule simply by $L(\lambda)$ from now on. To summarize, using the second part of Lemma 4.4 for the statement about $L(\lambda)^{*}$, we have shown:

Theorem 4.5 The supermodules $\{L(\lambda)\}_{\lambda \in X^{+}(T)}$ form a complete set of pairwise nonisomorphic irreducible G-supermodules. Moreover, for $\lambda \in X^{+}(T), L(\lambda)^{*} \cong L\left(-w_{0} \tilde{\lambda}\right)$.

\section{Remark 4.6}

(i) The second part of Theorem 4.5 implies that the restriction of the map $\sim$ from Theorem 4.3 gives a bijection $\sim: X^{+}(T) \rightarrow X^{+}(T)$.

(ii) A weight $\lambda=\sum_{i=1}^{m+n} \lambda_{i} \varepsilon_{i} \in X^{+}(T)$ is called restricted if either $p=0$ or $p>0$ and $\lambda_{i}-\lambda_{i+1}<p$ for each $i=1, \ldots, m-1, m+1, \ldots, m+n-1$. Assuming now that $p>0$, let $X^{+}(T)_{\text {res }}$ denote the set of all restricted $\lambda \in X^{+}(T)$. Let $F: G \rightarrow G_{\mathrm{ev}}$ be the Frobenius morphism defined on $g \in G(A)$ by raising all the matrix entries of $g$ to the power $p$, for each commutative superalgebra $A$. Let $G_{1}=\operatorname{ker} F$ be the Frobenius kernel. By a similar argument to $[5,6.4]$, the restriction of $L(\lambda)$ to $G_{1}$ remains irreducible for all $\lambda \in X^{+}(T)_{\text {res }}$, see [17].

(iii) Again for $p>0$, there is an analogue for $G$ of the Steinberg tensor product theorem. Given (ii), the proof is essentially the same as in [6, Section 9], see [17] for the details. To state the result, let $L_{\mathrm{ev}}(\lambda)$ denote the irreducible $G_{\mathrm{ev}}$-supermodule of highest weight $\lambda \in X^{+}(T)$ as in the proof of Lemma 4.4. Inflating through the Frobenius morphism $F: G \rightarrow G_{\mathrm{ev}}$, we obtain an irreducible $G$-supermodule $F^{*} L_{\mathrm{ev}}(\lambda) \cong L(p \lambda)$. In general, for $\lambda \in X^{+}(T)$, we can write $\lambda=\mu+p v$ where $\mu \in X^{+}(T)_{\text {res }}$ and $v \in X^{+}(T)$. Steinberg's tensor product theorem shows that

$$
L(\lambda) \cong L(\mu) \otimes F^{*} L_{\mathrm{ev}}(\nu) .
$$

(iv) Note for any $\lambda \in X^{+}(T), F^{*} L_{\mathrm{ev}}(\lambda)$ is trivial over $G_{1}$. So (ii), (iii) show in particular that $L(\lambda)$ is irreducible over $G_{1}$ if and only if $\lambda \in X^{+}(T)_{\text {res. }}$. Given this, the second part of Theorem 4.5 implies that the set $X^{+}(T)_{\text {res }}$ is stable under the map $\sim$. Finally, take $\lambda=\mu+p v$ where $\mu \in X^{+}(T)_{\text {res }}$ and $v \in X^{+}(T)$, as in (iii). Then,

$$
\begin{aligned}
L\left(-w_{0} \tilde{\lambda}\right) & \cong L(\lambda)^{*} \cong L(\mu)^{*} \otimes F^{*}\left(L_{\mathrm{ev}}(v)^{*}\right) \\
& \cong L\left(-w_{0} \tilde{\mu}\right) \otimes F^{*} L_{\mathrm{ev}}\left(-w_{0} v\right) \cong L\left(-w_{0}(\tilde{\mu}+p v)\right)
\end{aligned}
$$

Hence, $\tilde{\lambda}=\tilde{\mu}+p \nu$. This reduces the problem of computing $\tilde{\lambda}$ to the special case that $\lambda$ is restricted.

\section{Polynomial representations}

In this section, we discuss polynomial representations of $G$ in the spirit of Green's monograph [11]. Let $A(m \mid n)$ denote the subbialgebra $k[M a t]$ of $k[G]$, so $A(m \mid n)$ is the free 
commutative superalgebra on the generators $\left\{\tilde{T}_{i, j}\right\}_{1 \leq i, j \leq m+n}$ from (2.4). Obviously, $A(m \mid n)$ is $\mathbb{Z}$-graded by degree,

$$
A(m \mid n)=\bigoplus_{d \geq 0} A(m \mid n, d) .
$$

The subspace $A(m \mid n, d)$ is a finite dimensional subcoalgebra of $A(m \mid n)$. A representation $M$ of $G$ is called a polynomial representation (resp. a polynomial representation of degree $d$ ) if the comodule structure map $\eta: M \rightarrow M \otimes k[G]$ has image contained in $M \otimes A(m \mid n)$ (resp. in $M \otimes A(m \mid n, d))$. For example, the $d$ th tensor power $V^{\otimes d}$ of the natural representation of $G$ is polynomial of degree $d$. In general, a $G$-supermodule $M$ is polynomial of degree $d$ if it is isomorphic to a direct sum of subquotients of $V^{\otimes d}$.

By [7, Lemma 5.1], the decomposition (5.1) induces a decomposition of any polynomial representation into a direct sum of homogeneous polynomial representations. Moreover, the category of polynomial representations of degree $d$ is isomorphic to the category of supermodules over the Schur superalgebra

$$
S(m \mid n, d):=A(m \mid n, d)^{*},
$$

where the superalgebra structure on $S(m \mid n, d)$ is the one dual to the coalgebra structure on $A(m \mid n, d)$. Thus, the polynomial representation theory of $G$ reduces to studying representations of the finite dimensional superalgebras $S(m \mid n, d)$ for all $d \geq 0$. The latter has been investigated recently over a field of positive characteristic by Donkin [9], see also [21].

Let $I(m \mid n, d)$ denote the set of all functions from $\{1, \ldots, d\}$ to $\{1, \ldots, m+n\}$. We usually view $\boldsymbol{i} \in I(m \mid n, d)$ as a $d$-tuple $\left(i_{1}, \ldots, i_{d}\right)$ with entries in $\{1, \ldots, m+n\}$. In order to write down the various signs that will arise, introduce the notation $\epsilon_{i}=\left(\bar{i}_{1}, \ldots, \bar{i}_{d}\right) \in \mathbb{Z}_{2}^{d}$, for any $\boldsymbol{i} \in I(m \mid n, d)$. For tuples $\epsilon=\left(\epsilon_{1}, \ldots, \epsilon_{d}\right), \delta=\left(\delta_{1}, \ldots, \delta_{d}\right) \in \mathbb{Z}_{2}^{d}$ and $w \in S_{d}$, let

$$
\begin{gathered}
\alpha(\epsilon, \delta)=\prod_{1 \leq s<t \leq d}(-1)^{\delta_{s} \epsilon_{t}}, \\
\gamma(\epsilon, w)=\prod_{\substack{1 \leq s<t \leq d \\
w^{-1} s>w^{-1} t}}(-1)^{\epsilon_{\epsilon} \epsilon_{t}} .
\end{gathered}
$$

The symmetric group $S_{d}$ acts on the right on $I(m \mid n, d)$ by composition of functions, i.e. $\left(i_{1}, \ldots, i_{d}\right) \cdot w=\left(i_{w 1}, \ldots, i_{w d}\right)$. We will write $(\boldsymbol{i}, \boldsymbol{j}) \sim(\boldsymbol{k}, \boldsymbol{l})$ if $(\boldsymbol{i}, \boldsymbol{j})$ and $(\boldsymbol{k}, \boldsymbol{l})$ lie in the same orbit for the associated diagonal action of $S_{d}$ on $I(m \mid n, d) \times I(m \mid n, d)$. We say that a double index $(\boldsymbol{i}, \boldsymbol{j}) \in I(m \mid n, d) \times I(m \mid n, d)$ is strict if $\left(\bar{i}_{r}+\bar{j}_{r}\right)\left(\bar{i}_{s}+\bar{j}_{s}\right)=\overline{0}$ whenever $\left(i_{r}, j_{r}\right)=\left(i_{s}, j_{s}\right)$ for $1 \leq r<s \leq d$. Let $I^{2}(m \mid n, d)$ denote the set of all strict double indexes. Note $(\boldsymbol{i}, \boldsymbol{j})$ is strict if and only if the element

$$
\tilde{T}_{i, j}:=\tilde{T}_{i_{1}, j_{1}} \cdots \tilde{T}_{i_{d}, j_{d}} \in A(m \mid n, d)
$$

is non-zero. Moreover, if $\Omega(m \mid n, d)$ is a fixed set of orbit representatives for the action of $S_{d}$ on $I^{2}(m \mid n, d)$, then the elements $\left\{\tilde{T}_{i, j}\right\}_{(i, j) \in \Omega(m \mid n, d)}$ give a basis for $A(m \mid n, d)$. Given 
$(\boldsymbol{i}, \boldsymbol{j}),(\boldsymbol{k}, \boldsymbol{l}) \in I^{2}(m \mid n, d)$ with $(\boldsymbol{i}, \boldsymbol{j}) \sim(\boldsymbol{k}, \boldsymbol{l})$, we define a sign $\sigma(\boldsymbol{i}, \boldsymbol{j} ; \boldsymbol{k}, \boldsymbol{l})$ by

$$
\sigma(\boldsymbol{i}, \boldsymbol{j} ; \boldsymbol{k}, \boldsymbol{l})=\gamma\left(\epsilon_{\boldsymbol{i}}+\epsilon_{\boldsymbol{j}}, w\right)
$$

if $w$ is any element of $S_{d}$ with $(\boldsymbol{i}, \boldsymbol{j}) \cdot w=(\boldsymbol{k}, \boldsymbol{l})$. Note $\tilde{T}_{\boldsymbol{k}, \boldsymbol{l}}=\sigma(\boldsymbol{i}, \boldsymbol{j} ; \boldsymbol{k}, \boldsymbol{l}) \tilde{T}_{\boldsymbol{i}, \boldsymbol{j}}$

For $(\boldsymbol{i}, \boldsymbol{j}) \in I^{2}(m \mid n, d)$, let $\xi_{i, j} \in S(m \mid n, d)$ be the unique element satisfying

$$
\xi_{i, j}\left(\tilde{T}_{i, j}\right)=\alpha\left(\epsilon_{i}+\epsilon_{j}, \epsilon_{i}+\epsilon_{j}\right), \quad \xi_{i, j}\left(\tilde{T}_{k, l}\right)=0 \quad \text { for all }(\boldsymbol{k}, \boldsymbol{l}) \nsucc(\boldsymbol{i}, \boldsymbol{j})
$$

The elements $\left\{\xi_{i, j}\right\}_{(i, j) \in \Omega(m \mid n, d)}$ give a basis for $S(m \mid n, d)$. Given $\boldsymbol{i} \in I(m \mid n, d)$, let $v_{i}=v_{i_{1}} \otimes \cdots \otimes v_{i_{d}} \in V^{\otimes d}$, giving us a basis $\left\{v_{i}\right\}_{i \in I(m \mid n, d)}$ for the tensor space $V^{\otimes d}$. Since $V^{\otimes d}$ is a polynomial representation of degree $d$, there is a naturally induced representation

$$
\rho_{d}: S(m \mid n, d) \rightarrow \operatorname{End}_{k}\left(V^{\otimes d}\right) .
$$

Also let $e_{i, j}=e_{i_{1}, j_{1}} \otimes \cdots \otimes e_{i_{d}, j_{d}} \in \operatorname{End}_{k}(V)^{\otimes d} \cong \operatorname{End}_{k}\left(V^{\otimes d}\right)$, so $e_{i, j} v_{k}=\delta_{j, k} \alpha\left(\epsilon_{i}+\right.$ $\left.\epsilon_{\boldsymbol{j}}, \epsilon_{\boldsymbol{k}}\right) v_{\boldsymbol{i}}$ for $\boldsymbol{i}, \boldsymbol{j}, \boldsymbol{k} \in I(m \mid n, d)$.

Lemma 5.1 The representation $\rho_{d}: S(m \mid n, d) \rightarrow \operatorname{End}_{k}\left(V^{\otimes d}\right)$, is faithful and satisfies

$$
\rho_{d}\left(\xi_{i, j}\right)=\sum_{(\boldsymbol{k}, \boldsymbol{l}) \sim(\boldsymbol{i}, \boldsymbol{j})} \sigma(\boldsymbol{i}, \boldsymbol{j} ; \boldsymbol{k}, \boldsymbol{l}) e_{\boldsymbol{k}, \boldsymbol{l}}
$$

for each $(\boldsymbol{i}, \boldsymbol{j}) \in I^{2}(m \mid n, d)$. Moreover, for $(\boldsymbol{i}, \boldsymbol{j}),(\boldsymbol{k}, \boldsymbol{l}) \in I^{2}(m \mid n, d)$,

$$
\xi_{i, j} \xi_{k, l}=\sum_{(s, t) \in \Omega(m \mid n, d)} a_{i, j, k, l, s, t} \xi_{s, t}
$$

where $a_{\boldsymbol{i}, \boldsymbol{j}, \boldsymbol{k}, \boldsymbol{l}, \boldsymbol{s}, \boldsymbol{t}}=\sum \sigma(\boldsymbol{i}, \boldsymbol{j} ; \boldsymbol{s}, \boldsymbol{h}) \sigma(\boldsymbol{k}, \boldsymbol{l} ; \boldsymbol{h}, \boldsymbol{t}) \alpha\left(\epsilon_{\boldsymbol{h}}+\epsilon_{\boldsymbol{t}}, \epsilon_{\boldsymbol{s}}+\epsilon_{\boldsymbol{h}}\right)$, summing over all $\boldsymbol{h} \in$ $I(m \mid n, d)$ with $(\boldsymbol{s}, \boldsymbol{h}) \sim(\boldsymbol{i}, \boldsymbol{j}),(\boldsymbol{h}, \boldsymbol{t}) \sim(\boldsymbol{k}, \boldsymbol{l})$.

Proof: We first observe that the structure map $\eta: V^{\otimes d} \rightarrow V^{\otimes d} \otimes A(m \mid n, d)$ satisfies

$$
\eta\left(v_{j}\right)=\sum_{i \in I(m \mid n, d)}(-1)^{\bar{i}(\bar{i}+\bar{j})} \alpha\left(\epsilon_{i}+\epsilon_{j}, \epsilon_{i}\right) v_{i} \otimes \tilde{T}_{i, j}
$$

where $\overline{\boldsymbol{i}}=\bar{i}_{1}+\cdots+\bar{i}_{d}, \overline{\boldsymbol{j}}=\bar{j}_{1}+\cdots+\bar{j}_{d}$. Using this we calculate from the definition of the action:

$$
\begin{aligned}
\xi_{i, j} v_{\boldsymbol{l}} & =\left(1 \bar{\otimes} \xi_{\boldsymbol{i}, \boldsymbol{j}}\right) \sum_{\boldsymbol{k} \in I(m \mid n, d)}(-1)^{\overline{\boldsymbol{k}}(\overline{\boldsymbol{k}}+\bar{l})} \alpha\left(\epsilon_{\boldsymbol{k}}+\epsilon_{\boldsymbol{l}}, \epsilon_{\boldsymbol{k}}\right) v_{k} \otimes \tilde{T}_{\boldsymbol{k}, \boldsymbol{l}} \\
& =\sum_{\boldsymbol{k} \in I(m \mid n, d)} \alpha\left(\epsilon_{k}+\epsilon_{l}, \epsilon_{k}\right) \xi_{i, j}\left(\tilde{T}_{k, l}\right) v_{k} \\
& =\sum_{(\boldsymbol{k}, \boldsymbol{l}) \sim(\boldsymbol{i}, \boldsymbol{j})} \sigma(\boldsymbol{i}, \boldsymbol{j} ; \boldsymbol{k}, \boldsymbol{l}) \alpha\left(\epsilon_{\boldsymbol{k}}+\epsilon_{\boldsymbol{l}}, \epsilon_{\boldsymbol{l}}\right) v_{\boldsymbol{k}}
\end{aligned}
$$


This proves the formula for $\rho_{d}\left(\xi_{i, j}\right)$. Hence, $\rho_{d}$ is injective since the elements $\left\{\rho_{d}\left(\xi_{i, j}\right)\right\}_{(i, j) \in \Omega(m \mid n, d)}$ are clearly linearly independent. Finally, to derive the product rule, note that $e_{i, j} e_{k, l}=\delta_{j, k} \alpha\left(\epsilon_{i}, \epsilon_{j}, \epsilon_{k}+\epsilon_{l}\right) e_{i, l}$. Using this it is easy to compute the product $\xi_{i, j} \xi_{k, l}$ working in the representation $\rho_{d}$.

We next define a right action of the symmetric group $S_{d}$ on $V^{\otimes d}$ by letting

$$
v_{i}(j j+1)=(-1)^{\bar{i}_{j} \bar{i}_{j+1}} v_{i_{1}} \otimes \cdots \otimes v_{i_{j+1}} \otimes v_{i_{j}} \otimes \cdots \otimes v_{i_{d}}
$$

for each $\boldsymbol{i}=\left(i_{1}, \ldots, i_{d}\right) \in I(m \mid n, d)$ and each $1 \leq j<d$. For arbitrary $w \in S_{d}$, we have that

$$
v_{i} w=\gamma\left(\epsilon_{i}, w\right) v_{i \cdot w}
$$

Note the right action of $S_{d}$ is by even $G$-supermodule automorphisms, so it automatically commutes with the left action of $S(m \mid n, d)$ on $V^{\otimes d}$. The following theorem is well-known, see for example $[2,26]$.

Theorem $5.2 \rho_{d}: S(m \mid n, d) \rightarrow \operatorname{End}_{k S_{d}}\left(V^{\otimes d}\right)$ is an isomorphism.

Proof: We have already shown in Lemma 5.1 that $\rho_{d}$ is injective and that it maps $S(m \mid n, d)$ into $\operatorname{End}_{k S_{d}}\left(V^{\otimes d}\right)$. For surjectivity, take an arbitrary $\theta: V^{\otimes d} \rightarrow V^{\otimes d}$ commuting with the right action of $S_{d}$. Write

$$
\theta=\sum_{i, j \in I(m \mid n, d)} a_{i, j} e_{i, j}
$$

for some coefficients $a_{i, j}$. Since $\theta$ commutes with each $w \in S_{d}$, we have that $\left(\theta v_{j}\right) w=$ $\theta\left(v_{j} w\right)$. A computation using (5.8) gives that

$$
\gamma\left(\epsilon_{j}, w\right) \alpha\left(\epsilon_{i \cdot w}+\epsilon_{j \cdot w}, \epsilon_{j \cdot w}\right) a_{i \cdot w, j \cdot w}=\alpha\left(\epsilon_{i}+\epsilon_{j}, \epsilon_{j}\right) \gamma\left(\epsilon_{i}, w\right) a_{i, j}
$$

for each $\boldsymbol{i}, \boldsymbol{j}$. Simplifying this using the definitions of $\alpha$ and $\gamma$ then gives that

$$
a_{i \cdot w, j \cdot w}=a_{i, j} \gamma\left(\epsilon_{i}+\epsilon_{j}, w\right)
$$

Now note that if $(\boldsymbol{i}, \boldsymbol{j})$ is not strict, we can choose a transposition $w \in S_{d}$ so that $\boldsymbol{i} \cdot \boldsymbol{w}=$ $\boldsymbol{i}, \boldsymbol{j} \cdot w=\boldsymbol{j}$ and $\gamma\left(\epsilon_{\boldsymbol{i}}+\epsilon_{\boldsymbol{j}}, w\right)=-1$. Hence, $a_{\boldsymbol{i}, \boldsymbol{j}}=0$ in that case. Otherwise, if $(\boldsymbol{i}, \boldsymbol{j})$ is strict and $(\boldsymbol{k}, \boldsymbol{l}) \sim(\boldsymbol{i}, \boldsymbol{j})$, we have shown that $a_{\boldsymbol{k}, \boldsymbol{l}}=\sigma(\boldsymbol{i}, \boldsymbol{j} ; \boldsymbol{k}, \boldsymbol{l}) a_{\boldsymbol{i}, \boldsymbol{j}}$. It follows easily that $\theta$ is a linear combination of the elements $\rho_{d}\left(\xi_{i, j}\right)$ computed in Lemma 5.1. Hence, $\rho_{d}$ is onto.

We call a weight $\lambda=\sum_{i=1}^{m+n} \lambda_{i} \varepsilon_{i} \in X(T)$ a polynomial weight if $\lambda_{i} \geq 0$ for all $i$. Let $\Lambda(m \mid n, d)$ denote the set of all such polynomial weights satisfying in addition $\lambda_{1}+\cdots+$ $\lambda_{m+n}=d$. Note this is exactly the set of weights arising in the $G$-supermodule $V^{\otimes d}$. For 
$\boldsymbol{i} \in I(m \mid n, d)$, let $\operatorname{wt}(\boldsymbol{i}) \in \Lambda(m \mid n, d)$ denote the weight of the vector $v_{\boldsymbol{i}}$, so $\operatorname{wt}(\boldsymbol{i})=$ $\sum_{i=1}^{m+n} \lambda_{\boldsymbol{i}} \varepsilon_{\boldsymbol{i}}$ where there are $\lambda_{1} 1$ 's, $\lambda_{2} 2$ 's, $\ldots$ appearing in the tuple $\left(i_{1}, \ldots, i_{d}\right)$. Conversely, given $\lambda \in \Lambda(m \mid n, d)$, let $i_{\lambda}$ denote the tuple $(1, \ldots, 1,2, \ldots, 2, \ldots) \in I(m \mid n, d)$ with $\lambda_{i}$ $i$ 's for each $i$. Let

$$
\xi_{\lambda}=\xi_{i_{\lambda}, i_{\lambda}} \in S(m \mid n, d)
$$

We note that if $M$ is a polynomial representation of $G$ of degree $d$ then, by the argument in $[11,3.2]$, the subspace $\xi_{\lambda} M$ is exactly the $\lambda$-weight space $M_{\lambda}$ of $M$ as defined in (3.3). An elementary calculation using the product rule from Lemma 5.1 shows:

Lemma 5.3 For $(\boldsymbol{i}, \boldsymbol{j}) \in I^{2}(m \mid n, d)$,

$$
\xi_{\lambda} \xi_{i, j}=\left\{\begin{array}{ll}
\xi_{i, j} & \text { if } \operatorname{wt}(\boldsymbol{i})=\lambda, \\
0 & \text { otherwise },
\end{array} \quad \xi_{i, j} \xi_{\lambda}= \begin{cases}\xi_{i, j} & \text { if } \operatorname{wt}(\boldsymbol{j})=\lambda \\
0 & \text { otherwise }\end{cases}\right.
$$

In particular, $\left\{\xi_{\lambda}\right\}_{\lambda \in \Lambda(m \mid n, d)}$ is a set of mutally orthogonal even idempotents whose sum is the identity in $S(m \mid n, d)$.

Now we turn to the problem of classifying the irreducible polynomial representations of $G$, or equivalently, the irreducible $S(m \mid n, d)$-supermodules for all $d \geq 0$. Suppose for some $\lambda \in X^{+}(T)$ that the irreducible $G$-supermodule $L(\lambda)$ is a polynomial representation of degree $d$. Since all its weights are polynomial weights, $\lambda$ must belong to the set

$$
\Lambda^{+}(m \mid n, d):=\left\{\lambda \in \Lambda(m \mid n, d) \mid \lambda_{1} \geq \cdots \geq \lambda_{m}, \lambda_{m+1} \geq \cdots \geq \lambda_{m+n}\right\}
$$

of dominant polynomial weights of degree $d$. However,

$$
\Lambda^{++}(m \mid n, d):=\left\{\lambda \in \Lambda^{+}(m \mid n, d) \mid L(\lambda) \text { is a polynomial representation }\right\}
$$

will in general be a proper subset of $\Lambda^{+}(m \mid n, d)$, unlike the purely even case. The following lemma is an immediate consequence of Theorem 4.5 and the general remarks made at the beginning of the section.

Lemma 5.4 The supermodules $\{L(\lambda)\}_{\lambda \in \Lambda^{++}(m \mid n, d)}$ form a complete set of pairwise nonisomorphic irreducible $S(m \mid n, d)$-supermodules.

Of course the main problem now is to describe the set $\Lambda^{++}(m \mid n, d)$ combinatorially! Over fields of characteristic 0 , the answer is well-known, see [2] or [26]. In positive characteristic, Donkin has given a combinatorial description of $\Lambda^{++}(m \mid n, d)$ under the assumption that $d \leq m$, see [9, 2.3(4)]. We give here an alternative proof of Donkin's result, and describe $\Lambda^{++}(m \mid n, d)$ in general in Theorem 6.5 in the next section.

Theorem 5.5 Assume $d \leq m$. Then,

$$
\Lambda^{++}(m \mid n, d)=\left\{\lambda \in \Lambda^{+}(m \mid n, d) \mid \lambda_{m+1} \equiv \cdots \equiv \lambda_{m+n} \equiv 0(\bmod p)\right\} .
$$


Proof: We first recall the argument of Donkin from $[9,2.3(4)]$ to show that all $\lambda \in$ $\Lambda^{+}(m \mid n, d)$ with $\lambda_{m+1} \equiv \cdots \equiv \lambda_{m+n} \equiv 0(\bmod p)$ belong to $\Lambda^{++}(m \mid n, d)$, i.e. that $L(\lambda)$ is a polynomial representation for all such $\lambda$. Let $\omega_{i}=\varepsilon_{1}+\cdots+\varepsilon_{i}$. For any $r_{1}, \ldots, r_{m} \geq 0$, the polynomial representation

$$
V^{\otimes r_{1}} \otimes\left(\bigwedge^{2} V\right)^{\otimes r_{2}} \otimes \cdots \otimes\left(\bigwedge^{m} V\right)^{\otimes r_{m}}
$$

has unique highest weight $r_{1} \omega_{1}+\cdots+r_{m} \omega_{m}$. Hence $L\left(r_{1} \omega_{1}+\cdots+r_{m} \omega_{m}\right)$ is a composition factor of a polynomial representation, so polynomial. Now given an arbitrary $\lambda \in \Lambda^{++}(m \mid n, d)$ with $\lambda_{m+1} \equiv \cdots \equiv \lambda_{m+n} \equiv 0(\bmod p)$, we can write $\lambda=\mu+p v$ where $\mu \in X^{+}(T)_{\text {res }}$ is a restricted polynomial weight in the sense of Remark 4.6(ii) satisfying $\mu_{m+1}=\cdots=\mu_{m+n}=0$, and $v \in X^{+}(T)$ is an arbitrary polynomial weight. Then, the $G$-supermodule

$$
L(\mu) \otimes F^{*} L_{\mathrm{ev}}(v)
$$

has unique highest weight $\lambda$ so contains $L(\lambda)$ as a composition factor (actually it equals $L(\lambda)$ by Remark 4.6(iii), though we do not need this stronger result). Since $\mu$ can be expressed in the form $r_{1} \omega_{1}+\cdots+r_{m} \omega_{m}$ for $r_{i} \geq 0, L(\mu)$ is polynomial and $L_{\mathrm{ev}}(v)$, hence $F^{*} L_{\mathrm{ev}}(v)$, is polynomial by the classical theory. So $L(\lambda)$ is a polynomial representation, and $\lambda \in \Lambda^{++}(m \mid n, d)$.

Conversely, suppose that $\lambda \in \Lambda^{+}(m \mid n, d)$ has $\lambda_{m+i} \not \equiv 0(\bmod p)$ for some $1 \leq i \leq n$. Pick the minimal such $i$, and observe by the assumption $d \leq m$ that $\lambda_{m}=0$. Let $w \in D_{m, n}$ be the permutation $(m m+1 \ldots m+i)$, so

$$
\Phi_{w}^{+}=\Phi_{1}^{+}-\left\{\varepsilon_{m}-\varepsilon_{m+1}, \ldots, \varepsilon_{m}-\varepsilon_{m+i}\right\} \cup\left\{\varepsilon_{m+1}-\varepsilon_{m}, \ldots, \varepsilon_{m+i}-\varepsilon_{m}\right\} .
$$

Applying Lemma 4.2 using the sequence $\varepsilon_{m}-\varepsilon_{m+1}, \ldots, \varepsilon_{m}-\varepsilon_{m+i}$ of roots, we see that $L(\lambda) \cong L_{w}\left(\lambda-\varepsilon_{m}+\varepsilon_{m+i}\right)$. Thus, $\lambda-\varepsilon_{m}+\varepsilon_{m+i}$ is a weight of $L(\lambda)$. Since this is not a polynomial weight, $L(\lambda)$ cannot be a polynomial representation, i.e. $\lambda \notin \Lambda^{++}$ $(m \mid n, d)$.

We now explain how to descend from the Schur superalgebra $S(m \mid n, d)$ to the symmetric group $S_{d}$, assuming still that $d \leq m$. We need the following basic fact about functors defined by idempotents, cf. [11, 6.2] or [7, Corollary 2.13]. Recall that if $\xi \in S(m \mid n, d)$ is an even idempotent and $M$ is an $S(m \mid n, d)$-supermodule, we can view $\xi M$ naturally as a supermodule over the subalgebra $\xi S(m \mid n, d) \xi$ of $S(m \mid n, d)$.

Lemma 5.6 Let $\xi \in S(m \mid n, d)$ be an even idempotent. For $\lambda \in \Lambda^{++}(m \mid n, d), \xi L(\lambda)$ is either zero or it is an irreducible $\xi S(m \mid n, d) \xi$-supermodule. Moreover, the non-zero $\xi L(\lambda)$ 's give a complete set of pairwise non-isomorphic irreducible $\xi S(m \mid n, d) \xi$ supermodules.

One checks the following lemma using the product rule from Lemma 5.1. 
Lemma 5.7 Assume $d \leq m$ and let $\omega=\sum_{i=1}^{d} \varepsilon_{i} \in \Lambda(m \mid n, d)$. Then, for any $\boldsymbol{i} \in$ $I(m \mid n, d)$ and any $x \in S_{d}, \xi_{i, i_{\omega}} \xi_{\boldsymbol{i}_{\omega} \cdot x, i_{\omega}}=\gamma\left(\epsilon_{\boldsymbol{i}}, x\right) \xi_{\boldsymbol{i} \cdot x, \boldsymbol{i}_{\omega}}$.

Continue with $d \leq m$ and $\omega$ as in Lemma 5.7. By Lemma 5.3, the subalgebra $\xi_{\omega} S(m \mid n$, $d) \xi_{\omega}$ of $S(m \mid n, d)$ has basis $\left\{\xi_{i_{\omega} \cdot x, i_{\omega}}\right\}_{x \in S_{d}}$. Lemma 5.7 implies that the map

$$
k S_{d} \rightarrow \xi_{\omega} S(m \mid n, d) \xi_{\omega}, \quad x \mapsto \xi_{i_{\omega} \cdot x, i_{\omega}}
$$

is a superalgebra isomorphism. From now on, we will identify $k S_{d}$ with the subalgebra $\xi_{\omega} S(m \mid n, d) \xi_{\omega}$ of $S(m \mid n, d)$ in this way. Then, we can define the Schur functor

$$
f_{\omega}: S(m \mid n, d)-\bmod \rightarrow k S_{d}-\bmod
$$

On an $S(m \mid n, d)$-supermodule $M, f_{\omega} M$ is the $\omega$-weight space $\xi_{\omega} M$ of $M$ viewed as a $k S_{d}$-supermodule via the identification (5.10). On a morphism, the functor $f_{\omega}$ is defined by restriction.

\section{Remark 5.8}

(i) Recalling the definition of the action of $S_{d}$ on $V^{\otimes d}$ from (5.8), Lemma 5.7 also shows that the map $V^{\otimes d} \rightarrow S(m \mid n, d) \xi_{\omega}, v_{i} \mapsto \xi_{i, i_{\omega}}$ is an isomorphism of $S(m \mid n, d), k S_{d^{-}}$ bimodules.

(ii) An immediate consequence of (i) is that the Schur functor $f_{\omega}$ can be defined alternatively by $f_{\omega} M=\operatorname{Hom}_{G}\left(V^{\otimes d}, M\right)$ for a polynomial $G$-supermodule $M$ of degree $d$, where the $S_{d}$ action on $f_{\omega} M$ is induced by the natural right action of $S_{d}$ on $V^{\otimes d}$ from (5.7).

(iii) Another well-known consequence of (i) is the double centralizer property: for $d \leq m$, End $_{S(m \mid n, d)}\left(V^{\otimes d}\right)=k S_{d}$. Indeed, by (i) and properties of idempotents,

$$
\operatorname{End}_{S(m \mid n, d)}\left(V^{\otimes d}\right) \cong \operatorname{End}_{S(m \mid n, d)}\left(S(m \mid n, d) \xi_{\omega}\right) \cong \xi_{\omega} S(m \mid n, d) \xi_{\omega} \cong k S_{d} .
$$

One can extend this result to the case $d \leq m+n$ by similar arguments.

Recall now that a partition of $d$ is a sequence $\lambda=\left(\lambda_{1} \geq \lambda_{2} \geq \cdots\right)$ of non-negative integers satisfying $|\lambda|:=\lambda_{1}+\lambda_{2}+\cdots=d$. We usually identify $\lambda$ with its Young diagram

$$
\lambda=\left\{(i, j) \in \mathbb{Z}_{>0} \times \mathbb{Z}_{>0} \mid j \leq \lambda_{i}\right\}
$$

and refer to $(i, j) \in \lambda$ as the node in the $i$ th row and $j$ th column. We say that a partition $\lambda=\left(\lambda_{1} \geq \lambda_{2} \geq \cdots\right)$ is restricted if either $p=0$ or $p>0$ and $\lambda_{i}-\lambda_{i+1}<p$ for all $i=1,2, \ldots$ (cf. Remark 4.6(ii)). Let $\mathscr{P}(d)$ denote the set of all partitions of $d$, and $\mathscr{R} \mathscr{P}(d) \subseteq \mathscr{P}(d)$ denote the set of all restricted partitions of $d$.

Assuming still that $d \leq m$, we define an embedding

$$
x: \mathscr{R P}(d) \hookrightarrow \Lambda^{+}(m \mid n, d), \quad \lambda \mapsto \sum_{i=1}^{m} \lambda_{i} \varepsilon_{i} .
$$


By Theorem 5.5, we actually have that $x(\lambda) \in \Lambda^{++}(m \mid n, d)$ for $\lambda \in \mathscr{R} \mathscr{P}(d)$. So it makes sense to define

$$
D_{\lambda}=f_{\omega} L(x(\lambda))
$$

The following theorem shows in particular that the $D_{\lambda}$ 's are non-zero $k S_{d}$-modules.

Theorem 5.9 Assume $d \leq m$. For $\lambda \in \Lambda^{++}(m \mid n, d), f_{w} L(\lambda) \neq 0$ if and only if $\lambda=x(\mu)$ for some $\mu \in \mathscr{R} \mathscr{P}(d)$. Hence, the $k S_{d}$-modules $\left\{D_{\lambda}\right\}_{\lambda \in \mathscr{R} \mathscr{P}(d)}$ form a complete set of pairwise non-isomorphic irreducible $k S_{d}$-modules.

Proof: $\quad$ Take $\lambda \in \Lambda^{++}(m \mid n, d)$. By Theorem 5.5, if $\lambda \notin x(\mathscr{R} \mathscr{P}(d))$, we can decompose $\lambda=\mu+p v$ for polynomial weights $\mu, v \in X^{+}(T)$ with $\mu$ restricted in the sense of Remark 4.6(ii) and with $v \neq 0$. But then $L(\mu) \otimes F^{*} L(v)$ has unique highest weight $\lambda$, so has $L(\lambda)$ as a composition factor (actually it equals $L(\lambda)$ by Remark 4.6(iii)). Since the $\omega$-weight space of $L(\mu) \otimes F^{*} L(\nu)$ is zero, this shows that $f_{\omega} L(\lambda)=0$. Finally, by Lemma 5.6, the non-zero $f_{\omega} L(\lambda)$ with $\lambda \in \Lambda^{++}(m \mid n, d)$ must give a complete set of pairwise non-isomorphic irreducible $k S_{d}$-modules. It is well-known that the number of isomorphism classes of the latter is $|\mathscr{R} \mathscr{P}(d)|$, hence we must have that $f_{\omega} L(\lambda) \neq 0$ for all $\lambda \in x(\mathscr{R} \mathscr{P}(d))$. This completes the proof.

Remark 5.10 The $p$-regular partitions from the introduction are the conjugates of the restricted partitions. However, we will work from now on with the parametrization of the irreducible $k S_{d}$-modules by restricted partitions according to Theorem 5.9, though this is not the usual convention made in the literature. The relationship between our labeling and the standard labeling of James [13] is given by

$$
D_{\lambda} \cong D^{\lambda^{\prime}} \otimes \operatorname{sgn}
$$

One can see this as follows. Embedding $\Lambda(m, d):=\Lambda(m \mid 0, d)$ into $\Lambda(m \mid n, d)$ as the set of all weights with $\lambda_{m+1}=\cdots=\lambda_{m+n}=0$, let $\xi=\sum_{\lambda \in \Lambda(m, d)} \xi_{\lambda}$. Then, $\xi S(m \mid n, d) \xi$ can be identified with the classical Schur superalgebra $S(m, d):=S(m \mid 0, d)$ of [11], see the proof of Theorem 6.5 below for a similar construction. Moreover, given $\lambda \in$ $\Lambda^{+}(m, d):=\Lambda^{+}(m \mid 0, d), \xi L(\lambda)$ is the irreducible $S(m, d)$-module with highest weight $\lambda$. Notice that for $\omega$ as in Lemma 5.7, $\xi_{\omega} \xi=\xi_{\omega}$. Hence, our Schur functor $f_{\omega}$ from representations of $S(m \mid n, d)$ to representations of $k S_{d}$ factors through the Schur functor in [11, 6.4] from representations of $S(m, d)$ to representations of $k S_{d}$. So [11, 6.4] implies that $D_{\lambda}=L(x(\lambda)) \cong D^{\lambda^{\prime}} \otimes \operatorname{sgn}$ for each $\lambda \in \mathscr{R} \mathscr{P}(d)$.

\section{The Mullineux conjecture}

Let $\lambda \in \mathscr{P}(d)$ be a partition of $d$. We remind the reader that we choose to work with restricted partitions, hence the following definitions are the transpose of those usually found in the literature. The rim of $\lambda$ is defined to be the set of all nodes $(i, j) \in \lambda$ such 
that $(i+1, j+1) \notin \lambda$. The $p$-rim is a certain subset of the rim, defined as the union of the $p$-segments. The first $p$-segment is simply the first $p$ nodes of the rim, reading along the rim from left to right. The next $p$-segment is then obtained by reading off the next $p$ nodes of the rim, but starting from the column immediately to the right of the rightmost node of the first $p$-segment. The remaining $p$-segments are obtained by repeating this process. Of course, all but the last $p$-segment contain exactly $p$ nodes, while the last may contain less. For example, let $\lambda=\left(5,4,3^{2}, 1^{2}\right), p=5$. The nodes of the $p$-rim (which consists of two $p$-segments) are colored in black in the following picture.

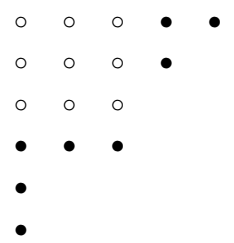

Let $a(\lambda)$ denote the number of nodes in the $p$-rim of $\lambda$.

We now define Mullineux conjugation

$$
\mathrm{M}: \mathscr{R} \mathscr{P}(d) \rightarrow \mathscr{R} \mathscr{P}(d)
$$

which is actually the transpose of the original definition from [22] since we are working with restricted rather than regular partitions. Given $\lambda \in \mathscr{R} \mathscr{P}(d)$, set $\lambda^{(1)}=\lambda$, and define $\lambda^{(i)}$ to be $\lambda^{(i-1)}-\left\{\right.$ the $p$-rim of $\left.\lambda^{(i-1)}\right\}$. Let $m$ be the largest number such that $\lambda^{(m)} \neq 0$. The Mullineux symbol of $\lambda$ is defined to be the array

$$
G(\lambda)=\left(\begin{array}{llll}
a_{1} & a_{2} & \cdots & a_{m} \\
r_{1} & r_{2} & \cdots & r_{m}
\end{array}\right)
$$

where $a_{i}=a\left(\lambda^{(i)}\right)$ is the number of the nodes in the $p$-rim of $\lambda^{(i)}$ and $r_{i}=\lambda_{1}^{(i)}$ is the first part of $\lambda^{(i)}$. The partition $\lambda$ can be uniquely reconstructed from its Mullineux symbol, see [22]. Now, $M(\lambda)$ is defined to be the unique restricted partition with

$$
G(\mathrm{M}(\lambda))=\left(\begin{array}{llll}
a_{1} & a_{2} & \cdots & a_{m} \\
s_{1} & s_{2} & \cdots & s_{m}
\end{array}\right)
$$

where

$$
s_{i}=\left\{\begin{array}{lll}
a_{i}-r_{i} & \text { if } a_{i} \equiv 0 & (\bmod p), \\
a_{i}+1-r_{i} & \text { if } a_{i} \not \equiv 0 & (\bmod p) .
\end{array}\right.
$$

Note in particular that the first part of $\mathrm{M}(\lambda)$ equals $s_{1}$.

As explained in the introduction, we will be concerned here with an equivalent formulation of the Mullineux algorithm discovered by $\mathrm{Xu}$ [28]. For $\lambda \in \mathscr{P}(d)$, let $\mathrm{J}(\lambda)$ be the partition 
obtained from $\lambda$ by deleting every node in the $p$-rim that is at the rightmost end of a row of $\lambda$ but that is not the $p$ th node of a $p$-segment. Let $j(\lambda)=|\lambda|-|\mathrm{J}(\lambda)|$ be the total number of nodes deleted. For example, with $\lambda=\left(5,4,3^{2}, 1^{2}\right), p=5$ as above, $\mathrm{J}(\lambda)$ is obtained by deleting the double-circled nodes:

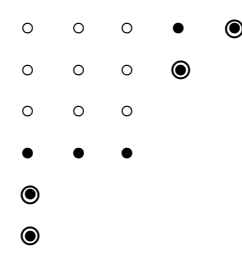

Hence $j(\lambda)=4$. Note the definitions of the maps $\mathrm{J}$ and $j$ make sense for arbitrary partitions, though to prove the Mullineux conjecture we only need to apply them to restricted partitions. In general, one has that $\mathrm{J}(\mu+p v)=\mathrm{J}(\mu)+p v$ and $j(\mu+p v)=j(\mu)$.

Recalling that $a(\lambda)$ is the number of nodes in the $p$-rim of $\lambda$, we note for arbitrary $\lambda \in \mathscr{P}(d)$ that

$$
j(\lambda)=\left\{\begin{array}{lll}
a(\lambda)-\lambda_{1} & \text { if } a(\lambda) \equiv 0 & (\bmod p), \\
a(\lambda)+1-\lambda_{1} & \text { if } a(\lambda) \not \equiv 0 & (\bmod p) .
\end{array}\right.
$$

Comparing with (6.1), this shows that for restricted $\lambda, j(\lambda)$ is the first part of the partition $\mathrm{M}(\lambda)$. More generally, it is proved in [4, Proposition 3.4] that for restricted $\lambda, \mathrm{M}(\mathrm{J}(\lambda))=$ $R(M(\lambda))$, where $R$ denotes first row removal. Using this fundamental fact, the following theorem of $\mathrm{Xu}$ [28] follows easily:

Theorem 6.1 For $\lambda \in \mathscr{R} \mathscr{P}(d), \mathrm{M}(\lambda)$ is the partition $\mu$ with $\mu_{i}=j\left(\mathrm{~J}^{i-1}(\lambda)\right)$.

We will refer to the algorithm for computing $\mathrm{M}(\lambda)$ given by Theorem 6.1 as Xu's algorithm. For an example, take $\lambda=\left(5,4,3^{2}, 1^{2}\right), p=5$ as above. Then $\mathrm{J}(\lambda)=\left(4,3^{3}\right), \mathrm{J}^{2}(\lambda)=$ $\left(3^{2}, 2^{2}\right), \mathrm{J}^{3}(\lambda)=\left(3^{2}, 1^{2}\right), \mathrm{J}^{4}(\lambda)=\left(3^{2}\right), \mathrm{J}^{5}(\lambda)=\left(2^{2}\right), \mathrm{J}^{6}(\lambda)=\left(1^{2}\right), \mathrm{J}^{7}(\lambda)=0$. Hence, $\mathrm{M}(\lambda)=\left(4,3,2^{5}\right)$.

We next explain the relationship between Xu's algorithm and Serganova's algorithm from Theorem 4.3. The main step is to prove the following alternative description of the map J.

Lemma 6.2 Suppose that $\lambda \in \mathscr{P}(d)$ with $\lambda_{m+1}=0$. Define $x_{1}, x_{2}, \ldots \in\{0,1\}$ by $x_{m+1}=x_{m+2}=\cdots=0$ and

$$
x_{i}=\left\{\begin{array}{lll}
1 & \text { if } \lambda_{i}+x_{i+1}+x_{i+2}+\cdots \not \equiv 0 & (\bmod p), \\
0 & \text { if } \lambda_{i}+x_{i+1}+x_{i+2}+\cdots \equiv 0 & (\bmod p),
\end{array}\right.
$$

for $i=m, m-1, \ldots, 1$. Then, $\mathrm{J}(\lambda)$ is the partition $\mu$ with $\mu_{i}=\lambda_{i}-x_{i}$.

Proof: Proceed by induction on $m$, the case $m=0$ being vacuous. For the induction step, take $\lambda \in \mathscr{R} \mathscr{P}(d)$ with $\lambda_{m+1}=0$. Define $x_{1}, x_{2}, \ldots$ and $\mu$ according to the statement of the 
lemma. By the induction hypothesis, we get that $\mathrm{J}(\mathrm{R}(\lambda))=\mathrm{R}(\mu)$, which shows in particular that $j(\mathrm{R}(\lambda))=|\mathrm{R}(\lambda)|-|\mathrm{R}(\mu)|=x_{2}+x_{3}+\cdots$. To complete the proof, it remains to show that the first part of $\mathrm{J}(\lambda)$ is equal to $\lambda_{1}-x_{1}$, or equivalently, $j(\lambda)=x_{1}+x_{2}+x_{3}+\cdots$.

If $a(\mathrm{R}(\lambda)) \equiv 0(\bmod p)$, then all the $p$-segments in the $p$-rim of $\mathrm{R}(\lambda)$ have $p$ nodes in them. Hence, the node $\left(1, \lambda_{2}\right)$ does not belong to the $p$-rim of $\lambda$. Using (6.2) for the second equality, we therefore get that

$$
a(\lambda)=\lambda_{1}-\lambda_{2}+a(\mathrm{R}(\lambda))=\lambda_{1}+j(\mathrm{R}(\lambda)) .
$$

Otherwise, if $a(\mathrm{R}(\lambda)) \neq \equiv 0(\bmod p)$, then the last $p$-segment of $\mathrm{R}(\lambda)$ has less than $p$ nodes in it. This implies that the node $\left(1, \lambda_{2}\right)$ must belong to the $p$-rim of $\lambda$, so

$$
a(\lambda)=\lambda_{1}-\lambda_{2}+1+a(\mathrm{R}(\lambda))=\lambda_{1}+j(\mathrm{R}(\lambda)) .
$$

Thus in either case, we have shown that

$$
a(\lambda)=\lambda_{1}+j(\mathrm{R}(\lambda))=\lambda_{1}+x_{2}+x_{3}+\cdots
$$

If this is zero $\bmod p$, then $x_{1}=0$ and $j(\lambda)=a(\lambda)-\lambda_{1}$ by (6.2). If it is non-zero mod $p$, then $x_{1}=1$ and $j(\lambda)=a(\lambda)+1-\lambda_{1}$. Either way, $j(\lambda)=x_{1}+x_{2}+\cdots$ as required.

Assume now that $m, n \geq d$. Recall the definition of the embedding $x: \mathscr{R} \mathscr{P}(d) \hookrightarrow$ $\Lambda^{+}(m \mid n, d)$ from (5.12). Instead, define

$$
y: \mathscr{R} \mathscr{P}(d) \hookrightarrow \Lambda^{+}(m \mid n, d), \quad \lambda \mapsto \sum_{i=1}^{n} \lambda_{i} \varepsilon_{m+i}
$$

Let $\sim: X^{+}(T) \rightarrow X^{+}(T)$ be the bijection defined combinatorially according to Theorem 4.3. Then:

Lemma 6.3 For $m, n \geq d$ and $\lambda \in \mathscr{R P P}(d), \widetilde{x(\lambda)}=y(\mathrm{M}(\lambda))$.

Proof: Compute $\widetilde{x(\lambda)}$ using Theorem 4.3 and the ordering $\varepsilon_{m}-\varepsilon_{m+1}, \ldots, \varepsilon_{1}-\varepsilon_{m+1} ; \varepsilon_{m}-$ $\varepsilon_{m+2}, \ldots, \varepsilon_{1}-\varepsilon_{m+2} ; \ldots ; \varepsilon_{m}-\varepsilon_{m+n}, \ldots, \varepsilon_{1}-\varepsilon_{m+n}$. After the first $m$ steps of the process, $x(\lambda)$ has been replaced by $x(\lambda)-\sum_{i=1}^{m} x_{i} \varepsilon_{i}+j(\lambda) \varepsilon_{m+1}$, where $x_{1}, \ldots, x_{m}$ are defined as in Lemma 6.2. Lemma 6.2 shows this is exactly the weight $x(\mathrm{~J}(\lambda))+j(\lambda) \varepsilon_{m+1}$. Repeating the argument for the next $m$ steps of Serganova's algorithm, we see similarly that $x(\mathrm{~J}(\lambda))+$ $j(\lambda) \varepsilon_{m+1}$ gets replaced by the weight $x(\mathrm{~J}(\mathrm{~J}(\lambda)))+j(\lambda) \varepsilon_{m+1}+j(\mathrm{~J}(\lambda)) \varepsilon_{m+2}$. Continuing in this way and using Xu's Theorem 6.1, we get after the $n m$ th step of Serganova's algorithm that $\widetilde{x(\lambda)}=y(\mathrm{M}(\lambda))$.

At last we are ready to prove the Mullineux conjecture, see also (5.14).

Theorem 6.4 For $\lambda \in \mathscr{R} \mathscr{P}(d), D_{\lambda} \otimes \operatorname{sgn} \cong D_{\mathrm{M}(\lambda)}$. 
Proof: Take $m=n \geq d$. Let $\sigma: G \rightarrow G$ be the supergroup automorphism defined for a commutative superalgebra $A$ and a matrix $g \in G(A)$ of the form (2.1) by

$$
\left(\begin{array}{c|c}
W & X \\
\hline Y & Z
\end{array}\right) \mapsto\left(\begin{array}{c|c}
Z & Y \\
\hline X & W
\end{array}\right)
$$

Given any $G$-supermodule $M$, we let $\sigma^{*} M$ denote the $G$-supermodule equal to $M$ as a vector superspace, but with new action defined by $g \cdot m=\sigma(g) m$ for all $g \in G(A), m \in M \otimes A$ and all commutative superalgebras $A$. In particular, $\sigma^{*}\left(V^{\otimes d}\right)$ denotes the tensor space $V^{\otimes d}$ with the action of $G$ twisted by $\sigma$ and with the original $S_{d}$-action from (5.7). We also write $V^{\otimes d} \otimes$ sgn for the $G$-supermodule $V^{\otimes d}$ but with the action of $S_{d}$ twisted by tensoring with sgn.

Let $\sigma:\{1, \ldots, 2 n\} \rightarrow\{1, \ldots, 2 n\}$ be the map $i \mapsto i+n$ if $i \leq n, i \mapsto i-n$ if $i \geq n+1$. Extend $\sigma$ to a map $\sigma: I(n \mid n, d) \rightarrow I(n \mid n, d)$ mapping $\boldsymbol{i}=\left(i_{1}, \ldots, i_{d}\right)$ to $\sigma(\boldsymbol{i})=\left(\sigma\left(i_{1}\right), \ldots, \sigma\left(i_{d}\right)\right)$. Define a map $\sigma: V \rightarrow \sigma^{*} V, v_{i} \mapsto v_{\sigma(i)}$. Obviously, this is an odd isomorphism of $G$-supermodules. Hence, the map

$$
\sigma^{\otimes d}: V^{\otimes d} \otimes \operatorname{sgn} \rightarrow \sigma^{*}\left(V^{\otimes d}\right), \quad v_{i} \otimes 1 \mapsto(-1)^{(d-1) \bar{i}_{1}+(d-2) \bar{i}_{2}+\cdots+\bar{i}_{d-1}} v_{\sigma(i)}
$$

is an isomorphism of $G$-supermodules. Using this formula, it is easy to check that the map $\sigma^{\otimes d}$ commutes with the action of a simple transposition $(j j+1) \in S_{d}$. Hence, $\sigma^{\otimes d}$ is an isomorphism of $G, S_{d}$-bimodules. It follows immediately that for any $G$-supermodule $M$, there is a natural isomorphism

$$
\operatorname{Hom}_{G}\left(V^{\otimes d}, \sigma^{*} M\right)=\operatorname{Hom}_{G}\left(\sigma^{*}\left(V^{\otimes d}\right), M\right) \cong \operatorname{Hom}_{G}\left(V^{\otimes d} \otimes \operatorname{sgn}, M\right)
$$

of $k S_{d}$-modules. Hence, recalling Remark 5.8(ii), we have a natural isomorphism

$$
\left(f_{\omega} M\right) \otimes \operatorname{sgn} \cong f_{\omega}\left(\sigma^{*} M\right)
$$

of $k S_{d}$-modules for any $S(n \mid n, d)$-supermodule $M$.

We now apply (6.4) to $M=L(x(\lambda))$. By (4.1) and Lemma 6.3,

$$
L(x(\lambda)) \cong L_{w_{1}}(\widetilde{x(\lambda)}) \cong L_{w_{1}}(y(\mathrm{M}(\lambda)))
$$

The automorphism $\sigma$ of $G$ swaps the Borel subgroups $B_{1}$ and $B_{w_{1}}$ and interchanges the two diagonal blocks in the torus $T$. Hence,

$$
\sigma^{*} L_{w_{1}}(y(\mathrm{M}(\lambda))) \cong L(x(\mathrm{M}(\lambda))) .
$$

So by (5.13) and (6.4), we get

$$
D_{\lambda} \otimes \operatorname{sgn}=\left(f_{\omega} L(x(\lambda))\right) \otimes \operatorname{sgn} \cong f_{\omega}\left(\sigma^{*} L(x(\lambda))\right) \cong f_{\omega} L(x(\mathrm{M}(\lambda)))=D_{\mathrm{M}(\lambda)} .
$$

This completes the proof. 
We conclude the article by completing the combinatorial description of the set $\Lambda^{++}$ $(m \mid n, d)$ that parametrizes the irreducible polynomial representations of $G$ of degree $d$ in Lemma 5.4. For $\lambda \in \Lambda^{+}(m \mid n, d)$, we will use the notation $t(\lambda)$ for the partition $\left(\lambda_{m+1}, \lambda_{m+2}, \ldots, \lambda_{m+n}\right)$, i.e. the "tail" of $\lambda$. Also recall the definition of $j$ from (6.2).

Theorem 6.5 For arbitrary $m, n, d$, we have that

$$
\Lambda^{++}(m \mid n, d)=\left\{\lambda \in \Lambda^{+}(m \mid n, d) \mid j(t(\lambda)) \leq \lambda_{m}\right\}
$$

Proof: Pick $M \geq m, N \geq n$ such that $M \geq d$ and $N=M-m$. Throughout the proof, we will identify $G L(m \mid n)$ with the closed subgroup of $G L(M \mid N)$ consisting (for each commutative superalgebra $A$ ) of all invertible matrices of the form

$$
\left(\begin{array}{cc|cc}
W & 0 & X & 0 \\
0 & I_{M-m} & 0 & 0 \\
\hline Y & 0 & Z & 0 \\
0 & 0 & 0 & I_{N-n}
\end{array}\right)
$$

where $W, X, Y, Z$ are as in (2.1). Embed $\Lambda(m \mid n, d)\left(\right.$ resp. $\left.\Lambda^{+}(m \mid n, d)\right)$ into $\Lambda(M \mid N, d)$ (resp. $\left.\Lambda^{+}(M \mid N, d)\right)$ as the set of all $\lambda$ with $\lambda_{m+1}=\cdots=\lambda_{M}=\lambda_{M+n+1}=\cdots=\lambda_{M+N}=$ 0 , and embed $I(m \mid n, d)$ into $I(M \mid N, d)$ as the set of all $d$-tuples $i$ with entries belonging to the set $\{1, \ldots, m, M+1, \ldots, M+n\}$. Let

$$
\xi=\sum_{\lambda \in \Lambda(m \mid n, d)} \xi_{\lambda} \in S(M \mid N, d) .
$$

The embedding $G L(m \mid n) \hookrightarrow G L(M \mid N)$ induces an isomorphism between the Schur superalgebra $S(m \mid n, d)$ and the subalgebra $\xi S(M \mid N, d) \xi$ of $S(M \mid N, d)$. The element $\xi_{i j}$ of $S(m \mid n, d)$ for $\boldsymbol{i}, \boldsymbol{j} \in I(m \mid n, d)$ corresponds to the element of $\xi S(M \mid N, d) \xi$ with the same name. We will denote the irreducible $S(M \mid N, d)$-supermodule of highest weight $\lambda \in \Lambda^{++}(M \mid N, d)$ by $L(\lambda)$, and the irreducible $S(m \mid n, d)$-supermodule of highest weight $\lambda \in \Lambda^{++}(m \mid n, d)$ by $L^{\prime}(\lambda)$.

Let $w \in D_{M, N}$ be the permutation

$$
w=\left(\begin{array}{cccc}
m+1 & m+2 & \cdots & M \\
M+1 & M+2 & \cdots & M+N
\end{array}\right)
$$

This defines a Borel subgroup $B_{w}$ of $G=G L(M \mid N)$, a set $\Phi_{w}^{+}$of positive roots and a dominance ordering $\leq_{w}$ on $X(T)$. Explicitly, for a commutative superalgebra $A, B_{w}(A)$ consists of all matrices in $G(A)$ of the form

$$
\left(\begin{array}{ll|l}
P & X & Y \\
0 & Q & 0 \\
\hline 0 & Z & R
\end{array}\right),
$$


where $P$ is an upper triangular $m \times m$ matrix, $Q, R$ are upper triangular $N \times N$ matrices, and $X, Y, Z$ are arbitrary. Like in (4.1), define a bijection $r: X(T) \rightarrow X(T)$ by the rule

$$
L_{1}(\lambda) \cong L_{w}(r(\lambda)) .
$$

The key observation is that if $\lambda$ is a weight $\notin \Lambda(m \mid n, d)$, then every $\mu \leq_{w} \lambda$ is also $\notin \Lambda(m \mid n, d)$. Hence, since the idempotent $\xi$ is just projection onto the weight spaces belonging to $\Lambda(m \mid n, d)$, we see that for $\lambda \in \Lambda^{++}(M \mid N, d), \xi L(\lambda) \cong \xi L_{w}(r(\lambda))$ is non-zero if and only if $r(\lambda) \in \Lambda^{+}(m \mid n, d)$. Moreover, in that case, $r(\lambda)$ is the highest weight of $\xi L(\lambda)$ with respect to the standard dominance ordering on $\Lambda(m \mid n, d)$. Viewing $\xi L(\lambda)$ as an $S(m \mid n, d)$-supermodule via the identification $S(m \mid n, d)=\xi S(M \mid N, d) \xi$, we have shown:

$$
\xi L(\lambda) \cong \begin{cases}L^{\prime}(r(\lambda)) & \text { if } r(\lambda) \in \Lambda^{+}(m \mid n, d) \\ 0 & \text { otherwise }\end{cases}
$$

Invoking Lemma 5.6, this means that

$$
\begin{aligned}
\Lambda^{++}(m \mid n, d) & =r\left(\Lambda^{++}(M \mid N, d)\right) \cap \Lambda^{+}(m \mid n, d) \\
& =\left\{\lambda \in \Lambda^{+}(m \mid n, d) \mid r^{-1}(\lambda) \in \Lambda^{++}(M \mid N, d)\right\} .
\end{aligned}
$$

Now we compute $r^{-1}(\lambda)$ for $\lambda \in \Lambda^{+}(m \mid n, d) \subseteq \Lambda^{+}(M \mid N, d)$. Let $t(\lambda)$ denote the partition $\left(\lambda_{M+1}, \lambda_{M+2}, \ldots, \lambda_{M+N}\right)$, i.e. the tail of $\lambda$ as in the statement of the theorem. Write $t(\lambda)=\mu+p v$ for partitions $\mu, v$ with $\mu$ restricted, so

$$
\lambda=\sum_{i=1}^{m} \lambda_{i} \varepsilon_{i}+\sum_{i=1}^{N} \mu_{i} \varepsilon_{M+i}+p \sum_{i=1}^{N} v_{i} \varepsilon_{M+i}
$$

Applying Lemma 4.2 repeatedly to the root sequence

$$
\begin{aligned}
& \varepsilon_{M+N}-\varepsilon_{m+1}, \ldots, \varepsilon_{M+1}-\varepsilon_{m+1} ; \varepsilon_{M+N}-\varepsilon_{m+2}, \ldots, \varepsilon_{M+1}-\varepsilon_{m+2} ; \ldots ; \\
& \varepsilon_{M+N}-\varepsilon_{M}, \ldots, \varepsilon_{M+1}-\varepsilon_{M} .
\end{aligned}
$$

and arguing as in the proof of Lemma 6.3 , one gets that

$$
r^{-1}(\lambda)=\sum_{i=1}^{m} \lambda_{i} \varepsilon_{i}+\sum_{i=1}^{N} j\left(\mathrm{~J}^{i-1}(\mu)\right) \varepsilon_{m+i}+p \sum_{i=1}^{N} \nu_{i} \varepsilon_{M+i}
$$

Note the number $j\left(\mathrm{~J}^{i-1}(\mu)\right)$ appearing here is simply the $i$ th part of $\mathrm{M}(\mu)$ according to Theorem 6.1. Finally, using Theorem 5.5 for the explicit description of $\Lambda^{++}(M \mid N, d)$, we deduce that $r^{-1}(\lambda)$ belongs to $\Lambda^{++}(M \mid N, d)$ if and only if $j(\mu) \leq \lambda_{m}$. Since $j(\mu)=$ $j(\mu+p v)=j(t(\lambda))$, this completes the proof. 


\section{Acknowledgments}

Research supported in part by the NSF (grant no. DMS-0139019). The idea that $G L(n \mid n)$ could be used to prove the Mullineux conjecture was inspired by a paper of A. Regev [24]. We are especially grateful to V. Serganova for explaining the material in Section 4 to us during a visit to the University of Oregon. We would also like to thank A. Kleshchev for pointing out the reference [4].

\section{References}

1. S. Ariki, "On the decomposition numbers of the Hecke algebra of type $G(m, 1, n)$," J. Math. Kyoto Univ. 36 (1996), 789-808.

2. A. Berele and A. Regev, "Hook Young diagrams with applications to combinatories and to representations of Lie superalgebras," Advances Math. 64 (1987), 118-175.

3. C. Bessenrodt and J. Olsson, "On residue symbols and the Mullineux conjecture," J. Alg. Comb. 7 (1998), $227-251$.

4. C. Bessenrodt, J. Olsson, and M. Xu, "On properties of the Mullineux map with an application to Schur modules," Math. Proc. Camb. Phil. Soc. 126 (1999), 443-459.

5. A. Borel, "Properties and linear representations of Chevalley groups," in Seminar on Algebraic Groups and Related Finite Groups, Lecture Notes in Mathematics, vol. 131, Springer, 1970.

6. J. Brundan and A. Kleshchev, "Modular representations of the supergroup Q(n), I," to appear in J. Algebra.

7. J. Brundan and A. Kleshchev, "Projective representations of symmetric groups via Sergeev duality," Math. Z. 239 (2002), 27-68.

8. S. Donkin, “On Schur algebras and related algebras, I,” J. Algebra 104 (1986), 310-328.

9. S. Donkin, "Symmetric and exterior powers, linear source modules and representations of Schur superalgebras," Proc. London Math. Soc. 83 (2001), 647-680.

10. B. Ford and A. Kleshchev, "A proof of the Mullineux conjecture," Math Z. 226 (1997), 267-308.

11. J.A. Green, Polynomial representations of $G L(n)$, Lecture Notes in Mathematics, vol. 830, Springer, 1980.

12. G.D. James, "The representation theory of the symmetric groups," Lecture Notes in Mathematics, vol. 682, Springer, 1978.

13. J.C. Jantzen, Representations of Algebraic Groups, Academic Press, 1987.

14. V. Kac, "Lie superalgebras," Advances Math. 26 (1977), 8-96.

15. V. Kac, "Representations of classical Lie superalgebras," in Lecture Notes in Mathematics, vol. 676, pp. 597-626, Springer, 1978.

16. A. Kleshchev, "Branching rules for modular representations of symmetric groups III," J. London Math. Soc. 54 (1996), 25-38.

17. J. Kujawa, "The representation theory of $G L(m \mid n)$," PhD thesis, University of Oregon, 2003.

18. A. Lascoux, B. Leclerc, and J.-Y. Thibon, "Hecke algebras at roots of unity and crystal bases of quantum affine algebras," Comm. Math. Phys. 181 (1996), 205-263.

19. D.A. Leites, "Introduction to the theory of supermanifolds," Russian Math. Surveys 35 (1980), 1-64.

20. Yu I. Manin, Gauge field theory and complex geometry, Grundlehren der mathematischen Wissenschaften 289, second edition, Springer, 1997.

21. N. Muir, "Polynomial representations of the general linear Lie superalgebras," PhD thesis, University of London, 1991.

22. G. Mullineux, "Bijections of $p$-regular partitions and $p$-modular irreducibles of symmetric groups," J. London Math. Soc. 20 (1979), 60-66.

23. I. Penkov and V. Serganova, "Representations of classical Lie superalgebras of type I," Indag. Math. (N.S.) 3 (1992), 419-466.

24. A. Regev, "Double centralizing theorems for the alternating groups," J. Algebra 250 (2002), 335-352.

25. V. Serganova, Automorphisms of complex simple Lie superalgebras and affine Kac-Moody algebras, $\mathrm{PhD}$ thesis, Leningrad State University, 1988. 
26. A. Sergeev, "Tensor algebra of the identity representation as a module over the Lie superalgebras $G L(n, m)$ and Q(n)," Math. USSR Sbornik 51 (1985), 419-427.

27. R. Steinberg, Lectures on Chevalley Groups, Yale University, 1967.

28. M. Xu, "On Mullineux' conjecture in the representation theory of symmetric groups," Comm. Algebra 25 (1997), 1797-1803.

29. M. Xu, "On p-series and the Mullineux conjecture," Comm. Algebra 27 (1999), 5255-5265. 